\title{
CONTINUOUS SUBJECTIVE EXPECTED UTILITY WITH NON-ADDITIVE PROBABILITIES*
}

\author{
Peter WAKKER \\ Netherlands Central Bureau of Statistics, 2270, AZ Voorburg, The Netherlands**
}

Submitted July 1987, accepted May 1988

\begin{abstract}
A well-known theorem of Debreu about additive representations of preferences is applied in a non-additive context, to characterize continuous subjective expected utility maximization for the case where the probability measures may be non-additive. The approach of this paper does not need the assumption that lotteries with known (objective) probability distributions over consequences are available.
\end{abstract}

\section{Introduction}

There is nowadays an increasing interest for the approach to decision making under uncertainty which explains violations of Savage's (1954) 'surething principle' by allowing for non-additivity of probability measures. A characterization of non-additivity of probability measures has been provided by Schmeidler (1984a, b, 1986). He assumed that not only consequences are available as result from a decision situation, but also all simple lotteries (with known probability distributions) over consequences. In many economic applications the introduction of lotteries with known probability distributions on consequences will be felt as an artificial construct. This motivated the present paper; it will remove the assumption of the availability of lotteries, and will replace that by the assumption of continuity of utility, an assumption which for most economic applications will not be felt as a restriction.

As a price for the greater generality obtained, the main characterizing condition, 'comonotonic cardinal coordinate independence', is not so simple and appealing as Schmeidler's main characterizing condition, 'comonotonic independence'. (In section 10 we shall sketch how the conditions in

*The views expressed in this paper are those of the author and do not necessarily reflect the policies of the Netherlands Central Bureau of Statistics. This paper is a rewritten version of Chapter VI of Wakker (1986). Part of the research, described in this paper, was done during a stay at the Tel Aviv University, Department of Economics, with financial support from the Netherlands Organization for the Advancement of Pure Research (Z.W.O.). The research topic of this paper was suggested to the author by D. Schmeidler. An anonymous referee gave helpful comments.

**Present address: Dept. of Mathematical Psychology, University of Nijmegen. 
Schmeidler's set-up, because of the presence of lotteries, do imply comonotonic cardinal coordinate independence.) A further price for the greater generality is that, if the main theorem is just 'one-step-more-complicated' than the result of Schmeidler, the proof of the main theorem will be far more complicated. The reason is that Schmeidler could use the lotteries on the set of certain acts to obtain immediately, through certainty equivalents, a representing function unique up to a positive affine transformation. This representing function was then characterized as the Choquet integral. In our set-up there is no easy way to obtain a representing function, unique up to a positive affine transformation; obtaining it will be the major complication in our proof. A restriction of this paper is that we only consider finite state spaces. The extension to arbitrary state spaces will be given in Wakker (1987). There also many applications of Schmeidler's approach will be given.

Schmeidler extends Anscombe and Aumann's (1963) derivation of subjective expected utility to non-additive probabilities. Analogously this paper extends Wakker (1984). Gilboa (1987) analogously extends Savage (1954).

The method of proof adopted in this paper shows how a well-known theorem of Debreu (1960), characterizing additive representations of preferences, can be of use in non-additive contexts. The techniques in section 7 may also be of use for the application of Debreu's theorem to subsets of Cartesian products. Some details in the proof have not been elaborated here; they can be found in Wakker (1986).

The expression 'non-additive probability' is used in an informal way in this paper; the formal term will be 'capacity', see Definition 2.1 .

Let us finally note that the main result, Theorem 5.1, can be understood without consultation of the remainder of the text, with the exception of some definitions, listed directly above that theorem.

\section{The preference relation}

By $S=\{1, \ldots, n\}$ we denote the set of states (of nature). $\Gamma$ denotes a set of consequences. Throughout this paper we assume that $\Gamma$ is a connected separable topological space. Acts are elements of the Cartesian product $\Gamma^{n}$, or, equivalently, functions from the set of states to the set of consequences, with act $f$ assigning consequence $f_{i}$ to state $i$ for all $i$. The Cartesian product $\Gamma^{n}$ is endowed with the product topology. By $\geqslant$ the preference relation of a decision maker on the set of acts is denoted. For any $\alpha$ in $\Gamma, \bar{\alpha}$ is the constant act assigning consequence $\alpha$ to every state. For $A \subset S$, and $f \in \Gamma^{n}, f_{A}$ denotes the restriction of $f$ to $A$. This can be considered to be an element of $\prod_{i \in A} \Gamma$. For $g \in \Gamma^{n}, f_{-A} g_{A}$ is $f_{A^{c}} g_{A}$, i.e., the act which equals $g$ on $A$, and $f$ on $A^{c}$. Analogous notations are, for $1 \leqq i \neq j \leqq n, \alpha, \beta \in \Gamma, f \in \Gamma^{n}$ :

$$
f_{-i} \alpha \text { is }\left[f \text { with } f_{i} \text { replaced by } \alpha\right] \text {, }
$$


and

$$
f_{-i, j} \alpha, \beta \text { is }\left[f \text { with } f_{i} \text { replaced by } \alpha \text { and } f_{j} \text { by } \beta\right] \text {. }
$$

The binary relation $\geqslant$ is a weak order on a subset $E$ of $\Gamma^{n}$ if it is complete on $E$ (i.e., for all $f, g$ in $E: f \geqslant g$ or $g \geqslant f$ ) and transitive on $E$ (i.e., for all $f, g, h$ in $E$ : if $f \geqslant g$ and $g \geqslant h$ then $f \geqslant h$ ). We write $f>g$ if $f \geqslant g$ and not $g \geqslant f, f \approx g$ if $f \geqslant g$ and $g \geqslant f, f \preccurlyeq g$ if $g \geqslant f$, and $f<g$ if $g \succ f$. We call $\geqslant$ trivial on $E \subset \Gamma^{n}$ if $f \approx g$ for all $f, g$ in $E$. By $\geqslant$ we also denote the binary relation on $\Gamma$ defined by $\alpha \geqslant \beta$ if $\bar{\alpha} \geqslant \bar{\beta}$. In the terminologies above, and below, 'on $\Gamma^{n}$ ' will often bc omitted. Further $\geqslant$ is continuous if $\left\{f \in \Gamma^{n}: f \geqslant g\right\}$ and $\left\{f \in \Gamma^{n}: g \geqslant f\right\}$ are closed for all $g \in \Gamma^{n}$.

Let again $E \subset \Gamma^{n}$. A function $\phi: E \rightarrow \mathbb{R}$ represents $\geqslant$ on $E$ if, for all $f, g$ in $E$, $f \geqslant g$ iff $\phi(f) \geqq \phi(g)$. We use the term 'increasing' rather than 'strictly increasing'. A positive affine transformation adds a real number, next multiplies with a positive real number.

Definition 2.1. A function $v: 2^{S} \rightarrow \mathbb{R}$ is a capacity if

$$
\begin{aligned}
& v(\varnothing)=0, \\
& v(S)=1, \\
& A \subset B \Rightarrow v(A) \leqq v(B) \text { (monotonicity). }
\end{aligned}
$$

Definition 2.2. Let $v: 2^{S} \rightarrow \mathbb{R}$ be a capacity. Then, for any function $\phi: S \rightarrow \mathbb{R}$, the Choquet integral of $\phi$ with respect to $v$, denoted as $\int_{S} \phi \mathrm{d} v$, or as $\int \phi \mathrm{d} v$, is

$$
\int_{0}^{\infty} v(\{i \in S: \phi(i) \geqq \tau\}) \mathrm{d} \tau+\int_{-\infty}^{0}[v(\{i \in S: \phi(i) \geqq \tau\})-1] \mathrm{d} \tau .
$$

For additive capacities the Choquet integral coincides with the usual integral, as follows from integration by parts. Let $\pi$ be a permutation on $\{1, \ldots, n\}$ such that

$$
\phi(\pi(1)) \geqq \phi(\pi(2)) \geqq \cdots \geqq \phi(\pi(n)),
$$

and write for all $1 \leqq j \leqq n$,

$$
P^{\pi}(j):=v\left(\left\{i \in S: \pi^{-1}(i) \leqq \pi^{-1}(j)\right\}\right)-v\left(\left\{i \in S: \pi^{-1}(i)<\pi^{-1}(j)\right\}\right) .
$$

Now it can be seen that (2.4) equals 


$$
\sum_{j=1}^{n} P^{\pi}(j) \phi(j)
$$

We have

$$
\begin{aligned}
& \int \lambda \phi \mathrm{d} v=\lambda \int \phi \mathrm{d} v \text { for all } \lambda \geqq 0 \quad \text { (positive homogeneity), } \\
& \int(\lambda+\phi) \mathrm{d} v=\lambda+\int \phi \mathrm{d} v \text { for all } \lambda \in \mathbb{R} \quad \text { (translation invariance). }
\end{aligned}
$$

Further,

$$
\text { if } \phi(i) \geqq \phi^{*}(i) \text { for all } i \text {, then } \int \phi \mathrm{d} v \geqq \int \phi^{*} \mathrm{~d} v \quad \text { (monotonicity). }
$$

If we consider the Choquet integral as a function(al) from $\mathbb{R}^{n}$ to $\mathbb{R}$, with $\left(\lambda_{1}, \ldots, \lambda_{n}\right) \in \mathbb{R}^{n}$ interpreted as the function assigning $\lambda_{j}$ to every $j$, then:

Proposition 2.1. The Choquet-integral is continuous.

Proof. See Schmeidler (1984a), or Wakker (1986, Proposition VI.2.4).

We need the following (2.11) in the proof of Theorem 8.1. Note that the $P^{\pi}(j)$ of (2.6) uniquely determine $v$ : for any $A \subset S$, take a $\pi$ such that $A=\{\pi(1), \ldots, \pi(i)\}$; then $v(A)=\sum_{j=1}^{i} P^{\pi}(\pi(j))$. This also shows that if one takes an arbitrary collection of real numbers $P^{\pi}(j)$, one $P^{\pi}(j)$ for every $1 \leqq j \leqq n$ and permutation $\pi$ on $\{1, \ldots, n\}$, then there exists a (necessarily unique) capacity $v$ such that any $P^{\pi}(j)$ can be derived from $v$ as in (2.6) if and only if for all $i, \pi, \pi^{\prime}$ :

$$
\begin{aligned}
& P^{\pi}(i) \geqq 0 ; \sum_{j=1}^{n} P^{\pi}(j)=1 \\
& {\left[\{\pi(1), \ldots, \pi(i)\}=\left\{\pi^{\prime}(1), \ldots, \pi^{\prime}(i)\right\}\right] \Rightarrow\left[\sum_{j=1}^{i} P^{\pi}(\pi(j))=\sum_{j=1}^{i} P^{\pi^{\prime}}\left(\pi^{\prime}(j)\right)\right]}
\end{aligned}
$$

\section{Comonotonicity}

The following is a central notion:

Definition 3.1. A set $C \subset \Gamma^{n}$ is comonotonic if no $f, g \in C, i, j \in S$ exist such that simultaneously $f_{i} \succ f_{j}$ and $g_{j} \succ g_{i}$.

Definition 3.2. For a permutation $\pi$ on $S, C^{\pi}:=\left\{f \in \Gamma^{n}: f_{\pi(1)} \geqslant f_{\pi(2)} \geqslant \cdots \geqslant\right.$ $\left.f_{\pi(n)}\right\} . C^{i d}=C^{\pi}$ with $\pi$ identity. 
For $f \in \Gamma^{n}, \succcurlyeq_{f}$ is the binary relation on $S$ defined by $i \succcurlyeq_{f} j$ whenever $f_{i} \succcurlyeq f_{j}$. If $\geqslant$ is a weak order, then so is $\succcurlyeq_{f}$. Further, for $S \subset \Gamma^{n}, \succcurlyeq_{s}:=$ $\bigcap_{f \in S} \geqslant_{s}$. Thus $i \succcurlyeq_{s} j$ iff $f_{i} \succcurlyeq_{j}$ for all $f \in S$. With an ordering a weak order for which no different elements are equivalent, we obtain

Lemma 3.1. Let $S \subset \Gamma^{n}$. Let $\geqslant$ be a weak order. The following three statements are equivalent:

(i) $S$ is comonotonic.

(ii) $\succcurlyeq_{s}$ is a weak order.

(iii) $S \subset C^{\pi}$ for some permutation $\pi$ on $S$.

Proof. (iii) follows from (ii) by letting $\pi$ be such that $i>_{s} j \Rightarrow \pi^{-1}(i)<\pi^{-1}(j)$. If (iii) holds, then for $f, g \in S, f_{i}>f_{j}$ and $g_{j}>g_{i}$ would imply $\pi^{-1}(i)<\pi(j)$ and $\pi^{-1}(j)<\pi^{-1}(i)$, which cannot hold. So (i) follows. Finally, (i) is assumed, and (ii) is derived. Transitivity of $\succcurlyeq_{\mathrm{s}}$ is by transitivity of $\succcurlyeq$. So completeness of $\geqslant_{\mathrm{s}}$ remains to be derived. If not $j \geqslant_{s} i$, then there is $f \in S$ with $f_{i}>f_{j}$. By comonotonicity $g_{i} \gtrless_{j}$ for all $g \in S$. So $i \succcurlyeq_{s} j$.

Statements (ii) and (iii) in Lemma 3.1 were observed by Schmeidler. Because of these results he called the acts in $S$ 'common monotonic', subsequently abbreviated to 'comonotonic'. Dellacherie (1970) may have been the first to see the importance of comonotonicity in connection with the Choquet integral.

Definition 3.3. Let $C \subset \Gamma^{n}$. Then state $i$ is inessential (with respect to $\geqslant$ ) on $C$ if $h_{-i} \alpha \approx h_{-i} \beta$ for all $h_{-i} \alpha, h_{-i} \beta \in C$. If $i$ is inessential on $C^{\pi}$, then we also call $i \pi$-inessential. If $\pi$ is the identity, we write id-inessential. The opposite of 'inessential' always is essential.

A preparatory notation:

For $\alpha, \beta \in \Gamma, \alpha \vee \beta[$ respectively $\alpha \wedge \beta$ ] is $\alpha$ if $\alpha \geqslant \beta$

[respectively $\alpha \leqslant \beta], \beta$ otherwise.

Lemma 3.2. Let $\geqslant$ be a weak order. Let $f, g \in C^{\pi}$, and $f_{j}=g_{j}$ for all $\pi$-essential $j$. Then $f \approx g$.

Proof. Suppose $f, g \in C^{\text {id }}$. Define $f^{0}:=f, g^{0}:=g$, and inductively, for $j=$ $1, \ldots, n, f^{j}:=f_{-j}^{j-1}\left(f_{j} \vee g_{j}\right), g^{j}:=g_{-j}^{j-1}\left(f_{j} \vee g_{j}\right)$. Note that, for all id-essential $j, f_{j}=g_{j}=f_{j} \vee g_{j}$. Note also that, for all $j>1, f_{j} \vee g_{j} \preccurlyeq f_{j-1}^{j-1}, f_{j} \vee g_{j} \preccurlyeq g_{j-1}^{j-1}$ so that $f^{j}, g^{j} \in C^{\text {id }}$ for all $j$. We conclude: $f=f^{0} \approx f^{1} \approx \cdots \approx f^{n}=g^{n} \approx g^{n-1} \approx \cdots \approx$ $g^{0}=g$. 
Lemma 3.3. Let $\succcurlyeq$ be a weak order. Let $i$ be $\pi$-inessential for all $i, \pi$. Then $\succcurlyeq$ is trivial.

Proof. Let $f, g \in \Gamma^{n}$. Take any $\alpha \in C$. Since $\bar{\alpha} \in C^{\pi}$ for all $\pi$, there are $\pi, \pi^{\prime}$ such that $f, \bar{\alpha} \in C^{\pi}$ and $g, \bar{\alpha} \in C^{\pi^{\prime}}$. By the previous Lemma, $f \approx \bar{\alpha} \approx g$.

\section{Comonotonic cardinal coordinate independence}

The following definition adapts the condition of 'cardinal coordinate independence' of Wakker (1984) to the present non-additive context.

Definition 4.1. The binary relation $\succcurlyeq$ satisfies comonotonic cardinal coordinate independence (Com.CCI) if, for all permutations $\pi, \pi^{\prime}$ on $\{1, \ldots, n\}$, all $j$ and $\pi$-essential $i$, and all $f_{-i} \alpha, g_{-i} \beta, f_{-i} \gamma, g_{-i} \delta \in C^{\pi}$, and finally all $s_{-j} \alpha, t_{-j} \beta$, $s_{-j} \gamma, t_{-j} \delta \in C^{\pi^{\prime}}$ :

$$
\left[f_{-i} \alpha \preccurlyeq g_{-i} \beta \& f_{-i} \gamma \geqslant g_{-i} \delta \& s_{-j} \alpha \geqslant t_{-j} \beta\right] \Rightarrow\left[s_{-j} \gamma \geqslant t_{-j} \delta\right] .
$$

A way to gain insight into the condition is to consider the simple and elaborated proof in section 5, which may reveal the idea of strength of preference underlying the definition. Lemmas 4.1 and 4.3 will derive direct consequences of Com.CCI.

Assumption 4.1 (for this section). The binary relation $\geqslant$ is a weak order that satisfies Com.CCI.

Definition 4.2. The binary relation $\geqslant$ is coordinate independent $(C I)$ if, for all $i, f, g, \alpha, \beta$,

$$
\left[f_{-i} \alpha \geqslant g_{-i} \alpha\right] \Leftrightarrow\left[f_{-i} \beta \geqslant g_{-i} \beta\right] \text {. }
$$

It straightforwardly follows that, for weak orders, CI implies $\left[f_{-A} s_{A} \geqslant g_{-A} s_{A}\right] \Leftrightarrow\left[f_{-A} t_{A} \geqslant g_{-A} t_{A}\right]$. This we adapt to the present nonadditive context, by restricting it to comonotonic acts.

Definition 4.3. The binary relation $\geqslant$ satisfies comonotonic coordinate independence (Com.CI) if for all comonotonic $\left\{f_{-A_{A}} s_{A}, g_{-A} s_{A}, f_{-A} t_{A}, g_{-A} t_{A}\right\}$ we have $\left[f_{-A} s_{A} \geqslant g_{-A} s_{A}\right] \Leftrightarrow\left[f_{-A} t_{A} \geqslant g_{-A} t_{A}\right]$.

Lemma 4.1. The binary relation $\geqslant$ satisfies comonotonic coordinate independence.

Proof. First we consider the special case that $A$ contains one element, say $A=\{k\}$. Let $f_{-k} s_{k}, g_{-k} s_{k}, f_{-k} t_{k}, g_{-k} t_{k} \in C^{\pi}$. If $k$ is $\pi$-inessential, then $f_{-k} s_{k} \approx f_{-k} t_{k}$, and $g_{-k} s_{k} \approx g_{-k} t_{k}$, and everything follows. So let $k$ be $\pi$ - 
essential. Then $\left[f_{-k} s_{k} \preccurlyeq f_{-k} s_{k} \& f_{-k} t_{k} \geqslant f_{-k} t_{k} \& f_{-k} s_{k} \geqslant g_{-k} s_{k}\right]$ by Com.CCI imply $f_{-k} t_{k} \geqslant g_{-k} t_{k}$.

Next we consider the general case. Say $f_{-A} s_{A}, g_{-A} s_{A}, f_{-A_{A}} t_{A}, g_{-A_{A}} t_{A} \in C^{\text {id }}$. Define:

$$
a^{0}:=f_{-A} s_{A}, \quad b^{0}:=g_{-A} s_{A}, \quad c^{0}:=f_{-A} t_{A}, \quad d^{0}:=g_{-A} t_{A} .
$$

Then define, inductively, for $j=1, \ldots, n$ :

$$
\text { If } j \notin A \text {, then }\left(a^{j}, b^{j}, c^{j}, d^{j}\right):=\left(a^{j-1}, b^{j-1}, c^{j-1}, d^{j-1}\right) \text {. }
$$

If $j \in A$, then set $\alpha=s_{j} \vee t_{j}$ and define

$$
\left(a^{j}, b^{j}, c^{j}, d^{j}\right):=\left(a_{-j}^{j-1} \alpha, b_{-j}^{j-1} \alpha, c_{-j}^{j-1} \alpha, d_{-j}^{j-1} \alpha\right) .
$$

The above construction has been such that $a_{k}^{j}=c_{k}^{j}$ and $b_{k}^{j}=d_{k}^{j}$ for all $k \leqq j$, and such that all new acts are in $C^{\text {id }}$. For instance, if $j \in A$, then $a^{j-1}, b^{j-1}$, $c^{j-1}, d^{j-1} \in C^{\text {id }}$ imply, by simple manipulations, $\alpha \preccurlyeq a_{j-1}^{j}, \alpha \preccurlyeq b_{j-1}^{j}, \alpha \preccurlyeq c_{j-1}^{j}$, $\alpha \preccurlyeq d_{j-1}^{j}$. Further $a^{n}=c^{n}, b^{n}=d^{n}$. By repeated application of the already handled case where $A$ contains exactly one element, we conclude that each of the following preferences holds if and only if its predecessor and successor hold: $f_{-A} s_{A} \geqslant g_{-A} s_{A}, a^{0} \geqslant b^{0}, a^{1} \geqslant b^{1}, \ldots, a^{n} \geqslant b^{n}, c^{n} \geqslant d^{n}, c^{n-1} \geqslant d^{n-1}, \ldots, c^{0} \geqslant$ $d^{0}, f_{-A} t_{A} \geqslant g_{-A} t_{A}$.

Definition 4.4. We write $f_{A} \geqslant{ }_{A}^{\pi} g_{A}$ if: [there exists $s_{A^{c}}$ such that $f_{A^{2}} s_{A^{c}} \geqslant g_{A} s_{A^{c}}$, and $\left.f_{A} s_{A^{c}}, g_{A} s_{A^{c}} \in C^{\pi}\right]$.

Lemma 4.2. If $f_{A} \geqslant{ }_{A} g_{A}$, then $f_{A^{2}} s_{A^{c}} \geqslant g_{A} s_{A^{c}}$ for all $s_{A^{c}}$ for which $f_{A} s_{A^{c}}$, $g_{A} s_{A^{c}} \in C^{\pi}$.

\section{Proof. Direct from Lemma 4.1.}

Definition 4.5. The binary relation $\succcurlyeq$ satisfies weak monotonicity (w.mon.) if $f \geqslant g$ whenever $f_{i} \succcurlyeq g_{i}$ for all $i$. It satisfies comonotonic strong monotonicity (com.s.mon.) if $f>g$ for all comonotonic $\{f, g\} \subset C^{\pi}$ with $f_{i} \succcurlyeq g_{i}$ for all $i$ and $f_{i} \succ g_{i}$ for a $\pi$-essential $i$.

Lemma 4.3. The binary relation $\geqslant$ satisfies weak, and comonotonic strong, monotonicity.

Proof. First we derive weak monotonicity. In three steps:

Step 1. Let $g=f_{-k} \alpha$, let $\{f, g\}$ be comonotonic, say $f, g \in C^{\text {id }}$, and let $f_{k} \geqslant \alpha$. We show that $f \geqslant f_{-k} \alpha$.

Suppose $f<f_{-k} \alpha$. Contradiction will follow.

Define, for $j=0, \ldots, n$, 


$$
h^{j} \text { has } h_{1}^{j}=\cdots=h_{j}^{j}=f_{k}, \quad h_{j+1}^{j}=\cdots=h_{n}^{j}=\alpha .
$$

Then all $h^{j}$ are in $C^{\text {id }}$. By Com.CI, $f<f_{-k} \alpha$ implies $h_{-k}^{k} f_{k}<h_{-k}^{k} \alpha$, i.e., $h^{k}<h^{k-1}$. Id-essentially of $k$ follows. Thus, by Com.CCI,

$$
\begin{gathered}
{\left[h_{-k}^{k} \alpha \preccurlyeq h_{-k}^{k} \alpha \& h_{-k}^{k} \alpha \succcurlyeq h_{-k}^{k} f_{k} \& h_{-j}^{j} \alpha \succcurlyeq h_{-j}^{j} \alpha\right] \text { implies }} \\
h_{-j}^{j} \alpha \succcurlyeq h_{-j}^{j} f_{k}, \text { i.e., } h^{j-1} \succcurlyeq h^{j}, \text { for all } j \geqq 1 .
\end{gathered}
$$

Apparently $\bar{\alpha}=h^{0} \geqslant h^{1} \geqslant \cdots \geqslant h^{k-1}, h^{k-1}>h^{k}, h^{k} \geqslant \cdots \geqslant h^{n}=\bar{f}_{k}$. This, finally, contradicts $f_{k} \geqslant \alpha$. Step 1 is established.

Step 2. Let again $g=f_{-k} \alpha$, and $f_{k} \geqslant \alpha$. Now we do not assume that $\{f, g\}$ is comonotonic.

Let us assume that $f \in C^{\text {id }}$. We show that $f \geqslant f_{-k} \alpha$.

Let $l$ be such that $f_{l} \succcurlyeq \alpha, f_{j} \prec \alpha$ for all $j>l$. Then, by repeated application of the result of Step $1, f \geqslant f_{-k} f_{k+1} \geqslant f_{-k} f_{k+2} \geqslant \cdots \geqslant f_{-k} f_{l} \succcurlyeq f_{-k} \alpha$, since every two consecutive acts are comonotonic (e.g., $f_{-k} f_{k+2}$ and $f_{-k} f_{k+3}$ are in $C^{\pi}$ for a $\pi$ with $\pi(k+2)=k$ ). Step 2 is established.

Step 3. Now let $f_{i} \succcurlyeq g_{i}$ for all $i$; further $f$ and $g$ are general. We show that $f \geqslant g$.

By repeated application of the above result,

$$
f \geqslant f_{-1} g_{1} \geqslant\left(\left(f_{-1} g_{1}\right)_{-2} g_{2}\right) \geqslant \cdots \geqslant\left(\left(\left(f_{-1} g_{1}\right)_{-2} g_{2}\right) \cdots{ }_{n} g_{n}\right)=g .
$$

Weak monotonicity is established.

Next we derive com.s.mon. Suppose that $\{f, g\}$ is comonotonic, say $\{f, g\} \subset C^{\text {id }}$, and that $f_{j} \geqslant g_{j}$ for all $j$. Let further $f_{k} \succ g_{k}$ for an id-essential $k$. To derive is $f>g$. Define:

$h$ has $h_{j}=f_{j}$ for all $j \leqq k, \quad h_{j}=g_{j}$ for all $j>k$.

Both $(h=) h_{-k} f_{k}$ and $h_{-k} g_{k}$ are in $C^{\text {id }}$. By w.mon. $f \geqslant h_{-k} f_{k} \geqslant h_{-k} g_{k} \geqslant g$. It is sufficient for com.s.mon. to show that $h_{-k} f_{k}>h_{-k} g_{k}$. Suppose to the contrary that $h_{-k} f_{k} \preccurlyeq h_{-k} g_{k}$. We derive a contradiction.

Define $h^{0}, \ldots, h^{n}$ as in (4.1), with $\alpha=g_{k}$. Since $k$ is id-essential, by Com.CCI $\left[h_{-k}^{k} g_{k} \preccurlyeq h_{-k}^{k} g_{k} \& h_{-k}^{k} g_{k} \geqslant h_{-k}^{k} f_{k} \& h_{-j}^{j} g_{k} \geqslant h_{-j}^{j} g_{k}\right]$ implies $h_{-j}^{j} g_{k} \geqslant h_{-j}^{j} f_{k}$, i.e., $h^{j-1} \geqslant h^{j}$, for all $j \geqq 1$. So $\bar{g}_{k} \geqslant f_{k}$. This contradicts $f_{k}>g_{k}$.

Corollary 4.1. The binary relation $\geqslant$ is trivial iff $\alpha \geqslant \beta$ for all $\alpha, \beta \in \Gamma$.

Proof. If $\geqslant$ is trivial, then $\bar{\alpha} \geqslant \bar{\beta}$, so $\alpha \geqslant \beta$, for all $\alpha, \beta$. Next assume $\alpha \geqslant \beta$ for all $\alpha, \beta$. Then for any $f$ in any $C^{\pi}$, and any $\alpha \in \Gamma, f_{i} \succcurlyeq \bar{\alpha}_{i}$ for all $i$, and $\bar{\alpha} \in C^{\pi}$, hence by w.mon. $f \geqslant \bar{\alpha}$. Analogously $f \leqslant \bar{\alpha}$. So $f \approx \bar{\alpha}$. Also $f \approx \bar{\alpha} \approx g$ for all $f, g, \alpha: \geqslant$ is trivial.

Corollary 4.2. One $\pi$ has a $\pi$-essential state iff every $\pi$ has a $\pi$-essential state. 
Proof. If one $\pi$ has a $\pi$-essential state, then $\geqslant$ is not trivial. By Corollary $4.1 \bar{\alpha}<\bar{\beta}$ for some $\alpha, \beta \in \Gamma$. Since $\bar{\alpha}, \bar{\beta} \in C^{\pi}$ for every $\pi$, Lemma 3.2 implies that every $\pi$ has a $\pi$-essential state.

\section{The main theorem}

In this section we give the main theorem of this paper. After the theorem the simplest implication (i) $\Rightarrow$ (ii) in it is proved. The proof of (ii) $\Rightarrow$ (i), and of the uniqueness results, will be carried out in the following sections, and completed in section 9. A survey of the proof is given in section 10. The elementary definitions, needed to understand the theorem, can be found in the beginning of section 2 up to Definition 2.2. Further are needed Definitions 3.1, 3.2, 3.3, and 4.1.

Theorem 5.1 (main theorem). Let $n \in \mathbb{N}$. Let $\Gamma$ be a connected separable topological space. For the binary relation $\geqslant$ on $\Gamma^{n}$, the following two statements are equivalent:

(i) There exist a capacity $v$ on $2^{(1, \ldots, n\}}$ and a continuous function $U: \Gamma \rightarrow \mathbb{R}$ such that $f \mapsto \int(U \circ f) \mathrm{d} v$ represents $\geqslant$.

(ii) The binary relation $\geqslant$ is a continuous weak order that satisfies Com.CCI.

The following uniqueness results hold for $U, v$ of $(i)$ :

If some $\pi$ has two or more $\pi$-essential states, then $U$ is unique up to a positive affine transformation, and $v$ is uniquely determined.

If $\geqslant$ is not trivial, and no $\pi$ has two or more $\pi$-essential states, then $U$ is unique up to a continuous increasing transformation, and $v$ is uniquely determined.

If $\geqslant$ is trivial, then $U$ is any constant function, and $v$ is arbitrary.

Proof of (i) $\Rightarrow$ (ii) above. Suppose (i) holds. Obviously $\geqslant$ is a weak order. Further, the map $f \mapsto\left(U\left(f_{1}\right), \ldots, U\left(f_{n}\right)\right)$ is continuous, so is, by Proposition 2.1 , the map $\left(U\left(f_{1}\right), \ldots, U\left(f_{n}\right)\right) \mapsto \int(U \circ f) \mathrm{d} v$. Consequently the map $f \mapsto$ $\int(U \circ f) \mathrm{d} v$ is continuous. This implies continuity of $\geqslant$.

All that remains is Com.CCI. For this, first suppose that:

$$
\begin{aligned}
& i \text { is } \pi \text {-essential; } \quad f_{-i} \alpha \preccurlyeq g_{-i} \beta, \quad f_{-i} \gamma \geqslant g_{-i} \delta ; \\
& \left\{f_{-i} \alpha, g_{-i} \beta, f_{-i} \gamma, g_{-i} \delta\right\} \subset C^{\pi} .
\end{aligned}
$$


The two preferences give, by (2.7), with the $\pi$ in (2.7) identical to our present $\pi$ since $f_{i} \succcurlyeq f_{j} \Rightarrow U\left(f_{i}\right) \geqq U\left(f_{j}\right)$,

$$
\sum_{k \neq i} P^{\pi}(k) U\left(f_{k}\right)+P^{\pi}(i) U(\alpha) \leqq \sum_{k \neq i} P^{\pi}(k) U\left(g_{k}\right)+P^{\pi}(i) U(\beta)
$$

and

$$
\sum_{k \neq i} P^{\pi}(k) U\left(f_{k}\right)+P^{\pi}(i) U(\gamma) \geqq \sum_{k \neq i} P^{\pi}(k) U\left(g_{k}\right)+P^{\pi}(i) U(\delta)
$$

These two imply

$$
P^{\pi}(i)[U(\alpha)-U(\beta)] \leqq P^{\pi}(i)[U(\gamma)-U(\delta)] .
$$

Were $P^{\pi}(i)=0$, then by (2.7) and the representation of $\geqslant$ by $f \mapsto \int(U \circ f) \mathrm{d} v, i$ would be $\pi$-inessential. So

$$
P^{\pi}(i)>0
$$

The last two numbered results imply

$$
U(\alpha)-U(\beta) \leqq U(\gamma)-U(\delta) .
$$

Now suppose, besides (5.4), also

$$
s_{-j} \alpha \geqslant t_{-j} \beta ; \quad\left\{s_{-j} \alpha, t_{-j} \beta, s_{-j} \gamma, t_{-j} \delta\right\} \subset C^{\pi^{\prime}} .
$$

The preference implies: $\sum_{k \neq j} P^{\pi^{\prime}}(k) U\left(s_{k}\right)+P^{\pi^{\prime}}(j) U(\alpha) \geqq \sum_{k \neq j} P^{\pi^{\prime}}(k) U\left(t_{k}\right)+$ $P^{\pi^{\prime}}(j) U(\beta)$. This, and (5.7), imply

$$
\sum_{k \neq j} P^{\pi^{\prime}}(k) U\left(s_{k}\right)+P^{\pi^{\prime}}(j) U(\gamma) \geqq \sum_{k \neq j} P^{\pi^{\prime}}(k) U\left(t_{k}\right)+P^{\pi^{\prime}}(j) U(\delta) .
$$

Or: $s_{-j} \gamma \geqslant t_{-j} \delta$. This is exactly what, by Com.CCI, should follow from (5.4) and (5.8).

\section{Preparations for the proof}

Lemma 6.1. Let $E \subset \Gamma^{n}$ be open [respectively closed] with respect to the product topology on $\Gamma^{n}$. Let $A \subset S, h \in \Gamma^{n}$. Then the set $\left\{f_{A} \in \prod_{i \in A} \Gamma: f_{A} h_{A^{c}} \in E\right\}$ is open [respectively closed] w.r.t. the product topology on $\prod_{j \in A} \Gamma$.

Proof. Say $E$ is open. (For closed $E$, take complement.) Let $V$ be the set 
as defined above. Let $g_{A} \in V$. There exist open $E_{i}$, for all $i \in S$, such that $g_{A} h_{A^{c}}$ is in $\prod_{i \in S} E_{i}$, and such that the latter is a subset of $E$. We see that $g_{A} \in \prod_{i \in A} E_{i} \subset V . \prod_{i \in A} E_{i}$ is an open neighbourhood of $g_{A}$ within $V$.

Lemma 6.2. Let $\geqslant$ be a weak order on $\Gamma^{n}$, continuous with respect to the product topology. Then for all $f \in \Gamma^{n},\{\alpha \in \Gamma: \bar{\alpha}>f\}$ and $\{\alpha \in \Gamma: \bar{\alpha} \prec f\}$ are open subsets of $\Gamma$.

Proof. Let $\bar{\alpha}>f$. Then an open neighbourhood $V \subset \Gamma^{n}$ of $\bar{\alpha}$ exists such that $g>f$ for all $g \in V$. We may assume that $V$ is of the form $A_{1} \times \cdots \times A_{n}$, with all $A_{j}$ open subsets of $\Gamma$. Now $A:=\bigcap_{j=1}^{n} A_{j}$ gives an open neighbourhood of $\alpha$ within $\{\alpha \in \Gamma: \bar{\alpha}>f\}$. So the latter is open.

Analogously $\{\alpha \in \Gamma: \bar{\alpha} \prec f\}$ is open.

Lemma 6.3. Let no $\pi$ have two or more $\pi$-essential states. Let the assumptions in Theorem 5.1, and also (ii) there, hold. Then also (i) and the uniqueness results there hold. If $\geqslant$ is non-trivial, then $v$ only assigns values 0 and 1 .

Proof. If there is a $\pi$ with no $\pi$-essential state, then by Lemma 3.2, for all $\alpha, \beta \in \Gamma, \bar{\alpha} \approx \bar{\beta}$. By Corollary $4.1, \geqslant$ is trivial. Now (5.3), and (i), follow straightforwardly. So we assume:

Every $\pi$ has exactly one $\pi$-essential state.

The binary relation on $\Gamma$, also denoted by $\geqslant$, obviously is a weak order. By Lemma 6.2 it is continuous. By Debreu (1964) there exists a $\phi: \Gamma \rightarrow \mathbb{R}$, representing $\geqslant$ on $\Gamma$, unique up to a continuous increasing transformation. We can set $U:=\phi$, as we shall see; so any continuous increasing transform of $U$ can be used.

Next we define $v$. Let $A \subset S$ be arbitrary. By non-triviality we can take some $\alpha$ and $\beta$ such that $\alpha>\beta$. If $\bar{\alpha}_{A} \bar{\beta}_{A c}>\bar{\beta}$, then we define $v(A):=1$, otherwise $v(A):=0$. By com.s.mon. and Lemma 3.2, $v(A)=1$ iff for any $\pi$ with $\{\pi(1), \ldots, \pi(k)\}=A, A$ contains the $\pi$-essential state. This shows that $v$ is independent of the particular choice of $\alpha$ and $\beta$ above. Also it follows that $P^{\pi}(j)=0$ for all $\pi$-inessential $j$, and $P^{\pi}(j)=1$ for the $\pi$-essential $j$.

Now we show that with these constructions, $(i)$ in Theorem 5.1 holds. Let $f$ and $g$ be two acts. Let $f \in C^{\pi}, g \in C^{\pi^{\prime}}$. Let $i$ be the $\pi$-essential state, $j$ the $\pi^{\prime}$-essential state. Then, by Lemma 3.2, $f \approx \bar{f}_{i}, g \approx \bar{g}_{j}$. There now follows: $f \geqslant g \Leftrightarrow \bar{f}_{i} \succcurlyeq \bar{g}_{j} \Leftrightarrow U\left(f_{i}\right) \geqq U\left(g_{j}\right) \Leftrightarrow \sum P^{\pi}(k) U\left(f_{k}\right) \geqq \sum P^{\pi^{\prime}}(k) U\left(g_{k}\right) \Leftrightarrow \int(U \circ f) \mathrm{d} v \geqq$ $\int(U \circ g) \mathrm{d} v$.

Finally we derive the uniqueness result (5.2). We saw above that $U$ can be any continuous increasing transform of $\phi$. Since, obviously, $U$ has to 
represent $\geqslant$ on $\Gamma$, no other kind of $U$ can be taken: $U$ is unique up to a continuous increasing transformation.

For uniqueness of $v$ we consider an arbitrary $\pi$, and show that $P^{\pi}(i)=0$ for all $\pi$-inessential $i$. Then $P^{\pi}(j)$ must equal 1 for the $\pi$-essential $j$. These values $P^{\pi}(\cdot)$ uniquely determine $v$. So let, finally, $i=\pi(k)$ be $\pi$-inessential. Let $\alpha \succ \beta$. Let $f$ assign $\alpha$ to $\pi(1), \ldots, \pi(k), \beta$ to $\pi(k+1), \ldots, \pi(n)$. Then $f$ and $f_{-i} \beta$ are in $C^{\pi}$. By $\pi$-inessentiality of $i, f \approx f_{-i} \beta$. Since $U(\alpha)>U(\beta)$, by (2.7) we obtain $P^{\pi}(i)=0$.

Definition 6.1. The binary relation $\geqslant$ satisfies restricted solvability if, for all $i, f, g, \alpha, \gamma$

$$
\left[f_{-i} \alpha \geqslant g \geqslant f_{-i} \gamma\right] \Rightarrow\left[\text { there exists } \beta \text { such that } f_{-i} \beta \approx g\right] \text {. }
$$

Lemma 6.4. A continuous weak order $\geqslant$ satisfies restricted solvability.

Proof. See Krantz et al. (1971, section 6.12.3).

Definition 6.2. Let $C \subset \Gamma^{n}$. Let $\left(V_{j}\right)_{j=1}^{n}$ be an array of functions, each from a subset of $\Gamma$ to the reals. Then $\left(V_{j}\right)_{j=1}^{n}$ are additive value functions (for $\geqslant$ ) on $C$ if the function $V: f \mapsto \sum_{j=1}^{n} V_{j}\left(f_{j}\right)$ is well-defined on $C$, and represents $\geqslant$ on $C$. If a function $V$ on a subset $C$ of $\Gamma^{n}$ can be written as a sum $V: f \mapsto \sum_{j=1}^{n} V_{j}\left(f_{j}\right)$, then $V$ is additive.

Theorem 6.1 [Debreu (1960)]. Let at least three states be essential on $\Gamma^{n}$. The following two statements are equivalent for the binary relation $\geqslant$ on $\Gamma^{n}$ :

(i) There exists a continuous additive representation of the binary relation $\geqslant$.

(ii) The binary relation $\geqslant$ is a continuous weak order which satisfies $C I$.

Furthermore the function in (i) is unique up to a positive affine transformation.

Definition 6.3. $\alpha \in \Gamma$ is maximal [respectively minimal] if $\beta \succ \alpha$ [respectively $\beta<\alpha]$ for no $\beta \in \Gamma$.

\section{Additive value functions on $\boldsymbol{C}^{\mathrm{id}}$}

In this section we derive results for $C^{\text {id }}$. Of course, analogous results hold for any $C^{\pi}$.

Assumption 7.1 (for this section). The assumptions, and statement (ii) of Theorem 5.1 hold. There are at least two id-essential states. Further, we assume that all states are id-essential. No maximal or minimal consequences exist. 
The assumption of the existence of at least two id-essential states is essential for the sequel. The assumption that all states are id-essential is made only for convenience. By Lemma 3.2 id-inessential states do not affect the preference relation on $C^{\text {id }}$, and may just as well be suppressed from notation. They simply get assigned additive value functions $V_{j}^{\text {id }}$ that are constant, say zero. Let us, as a preparation, establish 'certainty equivalents'.

Lemma 7.1. For every $f \in \Gamma^{n}$ there exists $\alpha$ such that $f \approx \bar{\alpha}$.

Proof. For $f \in \Gamma^{n}$ there exist $i, j$, such that $f_{i} \geqslant f_{k} \geqslant f_{j}$ for all $k \in S$. Let $V:=\{\alpha \in \Gamma: \bar{\alpha}>f\}, W:=\{\beta \in \Gamma: f \succ \bar{\beta}\}$. Then $V \cap W=\phi . V$ and $W$ are open by Lemma 6.2. Now $f_{i} \notin W$ and $f_{i} \notin V$ by w.mon. From connectedness of $\Gamma$ existence can be derived of an $\alpha \notin V \cup W ; \bar{\alpha} \approx f$.

\subsection{Additive value functions $\left(V_{j}^{h}\right)_{j=1}^{n}$ on the sets $E^{h}$}

Notation 7.1. For $h \in C^{\mathrm{id}}, E^{h}:=E_{1}^{h} \times \cdots \times E_{n}^{h}$, with $E_{1}^{h}:=\left\{\alpha \in \Gamma: \alpha \geqslant h_{1}\right\}, E_{n}^{h}:=$

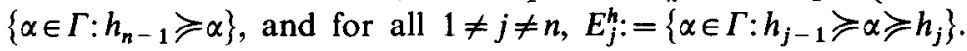

We have $h \in E^{h} \subset C^{\text {id }}$. The $E^{h \text { s }}$ are Cartesian products, and they are comonotonic so that on them the conditions of this paper hold without the comonotonicity premise. That enables us to apply, after some preparatory topological work, Theorem 3 of Debreu (1960).

Notation 7.2. The topology on $\Gamma$ is denoted as $\tau$. By $\tau(\geqslant)$ we denote the coarsest topology on $\Gamma$ with respect to which $\succcurlyeq$ on $\Gamma$ is continuous. By $\ldots \mid E$ we denote: 'restricted to $E$ '.

By Lemma 6.2 we see that $\tau(\geqslant)$ is coarser than $\tau$, so is connected and separable too. First let us note that:

Lemma 7.2. Any $E \subset \Gamma$ of the form $\{\alpha \in \Gamma: \sigma \geqslant \alpha \geqslant \tau\}, \quad\{\alpha \in \Gamma: \sigma \geqslant \alpha>\tau\}$, $\{\alpha \in \Gamma: \sigma \succ \alpha \geqslant \tau\},\{\alpha \in \Gamma: \sigma \succ \alpha \succ \tau\},\{\alpha \in \Gamma: \sigma \geqslant \alpha\},\{\alpha \in \Gamma: \sigma \succ \alpha\},\{\alpha \in \Gamma: \alpha \geqslant \tau\}$, or $\{\alpha \in \Gamma: \alpha \succ \tau\}$, is connected with respect to $\tau(\geqslant) \mid E$.

Proof. Throughout this proof, 'open' always refers to $\tau(\succcurlyeq)$. Let $E$ have a form as above. Let $F_{1}, F_{2}$ be open in $\Gamma$. Let $E_{1}=E \cap F_{1}, E_{2}=E \cap F_{2}$. Suppose $E_{1} \neq \phi \neq E_{2}, E_{1} \cap E_{2}=\phi, E_{1} \cup E_{2}=E$. We derive a contradiction.

Let $\alpha_{1} \in E_{1}, \alpha_{2} \in E_{2} . \tau(\geqslant)$ does not separate between $\approx$-equivalent consequences, so $\alpha_{1} \approx \alpha_{2}$ does not hold. Say $\alpha_{1} \prec \alpha_{2}$. Define:

$$
G_{1}:=\left[F_{1} \cap\left\{\alpha: \alpha_{1} \prec \alpha<\alpha_{2}\right\}\right] \cup\left[\left\{\alpha: \alpha \preccurlyeq \alpha_{1}\right\}\right], \text { and }
$$




$$
G_{2}:=\left[F_{2} \cap\left\{\alpha: \alpha_{1} \prec \alpha \prec \alpha_{2}\right\}\right] \cup\left[\left\{\alpha: \alpha \geqslant \alpha_{2}\right\}\right]
$$

Then $G_{1} \cap G_{2}=\phi, G_{1} \neq \phi \neq G_{2}$, and $G_{1} \cup G_{2}=\Gamma$ since $\left\{\alpha: \alpha_{1} \prec \alpha \prec \alpha_{2}\right\} \subset E$.

First we derive openness of $G_{1}$. For any element of $G_{1}$, an open neighbourhood $H$ of it within $G_{1}$ must be found. Let $\delta \in G_{1}$. If $\delta<\alpha_{1}$, take $H=\left\{\alpha: \alpha \prec \alpha_{1}\right\}$; if $\delta \succ \alpha_{1}, H=F_{1} \cap\left\{\alpha ; \alpha_{1} \prec \alpha \prec \alpha_{2}\right\}$ is taken. So finally let $\delta \approx \alpha_{1}$. There must be an open neighbourhood $H^{\prime}$ if $\delta$ within $F_{1}$ of the form $\{\alpha: \alpha>\mu\}$, or $\{\alpha: v>\alpha>\mu\}$, or $\{\alpha: v>\alpha\}$ for some $\mu, v \in \Gamma$. The first case is impossible since $\alpha_{2} \notin F_{1}$. So, finally, $H=\{\alpha: v>\alpha\}$ can be taken, in both remaining cases.

Analogously openness of $G_{2}$ is derived. Openness of $G_{1}$ and $G_{2}$ contradicts connectedness of $\Gamma$.

The above lemma shows that, if we use $\tau(\succcurlyeq)$ instead of $\tau$, then every $E_{j}^{h}$ is connected.

Lemma 7.3. Every $E_{j}^{h}$ is separable w.r.t. $\tau(\succcurlyeq) \mid E_{j}^{h}$.

Proof. This is obvious if $E_{j}^{h}$ contains exactly one $\approx$-equivalence class. If it contains more, then the intersection with $E_{j}^{h}$ of any countable dense subset of $\Gamma$ is dense in $E_{j}^{h}$, since every subset $H$ of $E_{j}^{h}$, open w.r.t. $\tau(\succcurlyeq) \mid E_{j}^{h}$, has open, and by connectedness non-empty, intersection with $H \cap\left\{\alpha: h_{j-1}>\alpha>h_{j}\right\}$.

Lemma 7.4. For any $h \in C^{\mathrm{id}}, \geqslant$, restricted to $E^{h}$, is continuous with respect to the product topology of the $\tau(\geqslant) \mid E_{j}^{h}$ 's.

Proof. Let $f, g \in E^{h}, f \succ g$. We construct an auxiliary $f^{*}$ such that $f^{*}>g$, and by means of this a subset $F_{1} \times \cdots \times F_{n}$ of $\left\{v \in E^{h}: v>g\right\}$, containing $f$, and with every $F_{j} \subset E_{j}^{h}$ open with respect to $\tau(\succcurlyeq) \mid E_{j}^{h}$. For the construction of $f_{1}^{*}$, consider

$$
V:=\left\{\alpha \in \Gamma:\left(\alpha, f_{2}, \ldots, f_{n}\right)>g\right\}
$$

By Lemma 6.1 this is open with respect to $\tau$. $V$ contains $f_{1}$ so is non-empty. If $V$ contains $h_{1}$, then $f_{1}^{*}=h_{1}$ and $F_{1}=E_{1}^{h}$ is taken. If $V$ does not contain $h_{1}$, then by connectedness of $\Gamma$ with respect to $\tau, V$ cannot be closed with respect to $\tau$, so not of the form $\left\{\alpha: \alpha \geqslant f_{1}\right\}$, as follows from Lemma 6.2. Since $V$, by w.mon., contains all $\alpha \geqslant f_{1}, V$ must contain an $\alpha \prec f_{1}$. This $\alpha$ cannot be $\preccurlyeq h_{1}$ (that, by w.mon., would imply $h_{1} \in V$ ). So $h_{1} \prec \alpha<f_{1}: \alpha \in E_{1}^{h}$. Take $f_{1}^{*}=\alpha, F_{1}=E_{1}^{h} \cap\{\beta \in \Gamma: \beta>\alpha\}$.

Anyway, $\left(f_{1}^{*}, f_{2}, \ldots, f_{n}\right) \succ g$, and $F_{1}$ is open with respect to $\tau(\succcurlyeq) \mid E_{1}^{h}$. By 
analogous constructions we obtain $f_{2}^{*}, F_{2}, \ldots, f_{n}^{*}, F_{n}$, such that $\left(f_{1}^{*}, f_{2}^{*}, \ldots, f_{j}^{*}, f_{j+1}, \ldots, f_{n}\right) \succ g$ for all $j, F_{j}=E_{j}^{h}$ if $f_{j}^{*}=h_{j}$, otherwise $h_{j} \prec f_{j}^{*} \prec$ $f_{j}$ and $F_{j}=E_{j}^{h} \cap\left\{\beta: \beta \succ f_{j}^{*}\right\}$. Finally, $\left(f_{1}^{*}, \ldots, f_{n}^{*}\right) \succ g$. For every $w \in F_{1} \times \cdots \times$ $F_{n}$, in particular $w=f, w_{j} \geqslant f_{j}^{*}$ for all $j$. By w.mon.: $w \geqslant f^{*}>g$.

So indeed, if $f \succ g$, we can construct $F_{1} \times \cdots \times F_{n} \subset\left[E^{h} \cap\{w: w \succ g\}\right]$, containing $f$, and open with respect to the product topology of $\tau(\geqslant) \mid E_{j}^{h}$, $j=1, \ldots, n$. Hence $\left\{f \in E^{h}: f \succ g\right\}$ is open with respect to the latter product topology, for all $g \in E^{h}$. Analogously $\left\{f \in E^{h}: f \prec g\right\}$ is open, for all $g$. Continuity of $\geqslant$ with respect to the product topology of the $\tau(\geqslant) \mid E_{j}^{h}$ 's follows.

Proposition 7.1. For any $h \in C^{\text {id }}$ there exists a continuous additive representation $V^{h}: f \mapsto \sum_{j=1}^{n} V_{j}^{h}\left(f_{j}\right)$ for $\geqslant$ on $E^{h}$, unique up to a positive affine transformation.

Proof. We only consider the case where three or more coordinates are essential on $E^{h}$; for the other case see Wakker (1986, Proposition VI.7.4). Since $E^{h}$, and any subset of it, is comonotonic, Com.CI holds without the comonotonicity restriction, i.e., CI holds.

Next we take care of the needed topological conditions. On every $E_{j}^{h}$ we take as topology $\tau(\geqslant) \mid E_{j}^{h}$. By Lemma $7.2, E_{j}^{h}$ is connected, by Lemma 7.3 it is separable. On $E^{h}$ we take the product topology. By Lemma 7.4, $\geqslant$ on $E^{h}$ is continuous with respect to this topology. By Theorem 3 of Debreu (1960) (a version of Theorem 6.1 where the factor sets are allowed to be different) there is an additive representation, unique up to a positive affine transformation, and continuous w.r.t. the product topology of $\tau(\succcurlyeq) \mid E_{j}^{h}(j=1, \ldots, h)$, so certainly w.r.t. the finer 'old' topology.

\subsection{Fitting the functions $V_{j}^{h}$ together on $C^{\text {id }}$}

Our next step is to show that there exist $V_{j}^{\text {id }}: \Gamma \rightarrow \mathbb{R}, j=1, \ldots, n$, such that for every $h$ and $j, V_{j}^{h}$ can be taken to be the restriction of $V_{j}^{\text {id }}$ to $E_{j}^{h}$. This of course could never be done if there were $A \subset S$, and $s, t \in C^{\text {id }}$, such that $\left(V_{j \in A}^{s}\right)_{j \in A}$ and $\left(V_{j}^{t}\right)_{j \in A}$ would be additive value functions for different binary relations on the 'common domain' $\prod_{j \in A}\left(E_{j}^{s} \cap E_{j}^{t}\right)$. By comonotonic coordinate independence (Lemma 4.1) that never happens. Both $\left(V_{j}^{s}\right) j \in A$ and $\left(V_{j}^{t}\right) j \in A$ are additive valuc functions for the binary relation $\geqslant_{A}^{\pi}$ with $\pi=$ identity, on appropriate domains.

Lemma 7.5. There exists a continuous additive function $V: f \mapsto \sum_{j=1}^{n} V_{j}\left(f_{j}\right)$ on $C^{\text {id }}$ which represents $\geqslant$ on every $E^{h}$, and which is unique up to a positive affine transformation. 
Proof. On every $E^{h}$ we are given an additive representation $V^{h}: f \mapsto$ $\sum_{j=1}^{n} V_{j}^{h}\left(f_{j}\right)$ which is unique up to a positive affine transformation. So we may add to every $V_{j}^{h}$ an arbitrary 'location' constant $\tau_{j}(h)$, and multiply the $V_{j}^{h}$ 's by one common positive 'scale constant' $\sigma(h)$, to obtain again an additive representation. The plan in the sequel is to choose, in five stages, scales and locations such that all $V_{j}^{\text {h's }}$ 'fit together', i.e., are the same on common domains. They can then be considered the restriction of one array $\left(V_{j}^{\text {id }}\right)_{j=1}^{n}$.

There must exist $\beta^{1}, \beta^{0} \in \Gamma$ such that $\beta^{1}>\beta^{0}$. We shall set $V_{j}^{\text {id }}\left(\beta^{0}\right)=0$ for all $j$, and $V_{1}^{\text {id }}\left(\beta^{1}\right)=1$. (In Assumption 8.1 the scale will be changed.)

Stage 1. Choice of scale and location on $E^{r}$ with $r=\bar{\beta}^{0}$.

Let $r$ ('reference point') $=\bar{\beta}^{0} . E_{1}^{r}=\left\{\alpha: \alpha \geqslant \beta^{0}\right\}$, it contains $\beta^{1} . E_{n}^{r}=\left\{\alpha: \alpha \preccurlyeq \beta^{0}\right\}$. For all $1 \neq j \neq n, E_{j}^{r}=\left\{\alpha: \alpha \approx \beta^{0}\right\}$. Of course we choose scale and locations such that

$$
V_{j}^{r}\left(\beta^{0}\right)=0 \quad \text { for all } j, \quad V_{1}^{r}\left(\beta^{1}\right)=1 .
$$

Stage 2. Choicc of scale on every $E^{h}$, and location for every $V_{1}^{h}, V_{n}^{h}$.

Let $h \in C^{\text {id }}$ be arbitrary. By Com.CI, $\left(V_{1}^{r}, V_{n}^{r}\right)$ and $\left(V_{1}^{h}, V_{n}^{h}\right)$ are additive value functions for the same $\geqslant_{\{1, n\}}^{\text {id }}$ on $\left(E_{1}^{r} \cap E_{1}^{h}\right) \times\left(E_{n}^{r} \cap E_{n}^{h}\right)$. Note that both 1 and $n$ are essential on $\left(E_{1}^{r} \cap E_{1}^{h}\right) \times\left(E_{n}^{r} \cap E_{n}^{h}\right)$ with respect to $\succcurlyeq_{\{1, n\}}^{\text {id }}$. By Lemmas 7.2 and 7.3, $E_{1}^{r} \cap E_{1}^{h}$ and $E_{n}^{r} \cap E_{n}^{h}$ are connected and separable with respect to the restrictions of $\tau(\geqslant)$, and by Proposition 7.1 we get uniqueness up to a positive affine transformation. So we can choose the scale for $\left(V_{1}^{h}, V_{n}^{h}\right)$, (and hence for all $\left(V_{j}^{h}\right)_{j=1}^{h}$ ) and the locations for $\left(V_{1}^{h}, V_{n}^{h}\right)$, such that $V_{1}^{r}=V_{1}^{h}$ on $E_{1}^{r} \cap E_{1}^{h}$, and $V_{n}^{r}=V_{n}^{h}$ on $E_{n}^{r} \cap E_{n}^{h}$. Thus we shall see, even stronger,

$$
V_{1}^{s}=V_{1}^{t} \text { and } V_{n}^{s}=V_{n}^{t} \text { on common domain for all } s, t \in C^{\text {id }} .
$$

This follows since, on $\left(E_{1}^{s} \cap E_{1}^{t}\right) \times\left(E_{n}^{s} \cap E_{n}^{t}\right),\left(V_{1}^{s}, V_{n}^{s}\right)$ and $\left(V_{1}^{t}, V_{n}^{t}\right)$ are additive value functions for the same $\geqslant_{[1, n\}}^{\text {id }}$, hence they can differ only with respect to their locations, and a common scale. However, for $j=1, n, V_{j}^{s}$ and $V_{j}^{t}$ coincide (with $V_{j}^{r}$ ) on $\left(E_{j}^{s} \cap E_{j}^{t} \cap E_{j}^{r}\right)$; hence they coincide on common domains.

Stage 3. Intermediate observation.

In fact, for all $s, t, j, V_{j}^{s}$ and $V_{j}^{t}$ now have the same scale, and differ only with respect to their location, as we shall show:

For all $s, t \in C^{\text {id }}$, and $1 \leqq j \leqq n$, there exist constants $\tau_{j}(s, t)$

such that on $E_{j}^{s} \cap E_{j}^{t}, V_{j}^{s}=\tau_{j}(s, t)+V_{j}^{t}$. 
For $j=1$ or $j=n$, by $(7.2)$, in fact $\tau_{j}(s, t)=0$. So let $1 \neq j \neq n$. Then $\left(V_{1}^{s}, V_{j}^{s}, V_{n}^{s}\right)$ and $\left(V_{1}^{t}, V_{j}^{t}, V_{n}^{t}\right)$ are additive value functions for the same $\geqslant_{\{1, j, n\}}^{\text {id }}$ on $\left(E_{1}^{s} \cap E_{1}^{t}\right) \times\left(E_{j}^{s} \cap E_{j}^{t}\right) \times\left(E_{n}^{s} \cap E_{n}^{t}\right)$. So they can differ only by location, and common scale. However, $V_{1}^{s}$ and $V_{1}^{t}$, and $V_{n}^{s}$ and $V_{n}^{t}$, coincide on their common domain (which contains more than one element). The common scales must be the same.

Stage 4. Choice of location for all $V_{j}^{h}$ s $(j \neq 1, n)$, having $\beta^{0}$ in their domain. Of course for all $V_{j}^{h}$ 's as above we choose location such that $V_{j}^{h}\left(\beta^{0}\right)=0$. Now not only (7.1) to (7.3) hold, but also:

If $V_{j}^{s}$ and $V_{1}^{t}$ have $\beta^{0}$ in their domain then they

coincide on common domain.

Stage 5. Choice of location for remaining $V_{j}^{h}$ 's.

Now let $h \in C^{\text {id }}$ and $j$ be such that $j \neq 1, j \neq n, \beta^{0} \notin E_{j}^{h}=\left\{\alpha: h_{j-1} \geqslant \alpha \geqslant h_{j}\right\}$. Say $h_{j} \succ \beta^{0}\left(h_{j-1} \prec \beta^{0}\right.$ is analogous $)$. Let $r(h) \in C^{\text {id }}$ be such that $(r(h))_{i}=h_{j-1}$ for all $i<j,(r(h))_{i}=\beta^{0}$ for all $i \geqq j$. Then $E_{j}^{h} \subset E_{j}^{r(h)}=\left\{\alpha: h_{j-1} \geqslant \alpha \geqslant \beta^{0}\right\}$. By Stage 4, $V_{j}^{r(h)}\left(\beta^{0}\right)=0$. We now choose the location of $V_{j}^{h}$ such that $V_{j}^{h}=V_{j}^{r(h)}$ on $E_{j}^{h}$. We shall show:

For all $s, t \in C^{\text {id }}, 1 \leqq j \leqq n, V_{j}^{s}$ and $V_{j}^{t}$ coincide on common domain.

We check this only for the case where $1 \neq j \neq n, \beta^{0}$ is neither in the domain of $V_{j}^{s}$, nor in that of $V_{j}^{t}$ (other cases have been dealt with before, or are analogous), and $s_{j} \succ \beta^{0}$. Here $E_{j}^{s}$ is of the form $\left\{\alpha: s_{j-1} \geqslant \alpha \geqslant s_{j}\right\}$. For $E_{j}^{t}$ to intersect $E_{j}^{s}$, we must have $t_{j} \succ \beta^{0}$. Now $V_{j}^{s}$ and $V_{j}^{r(s)}$ coincide on $E_{j}^{s} \cap E_{j}^{r(s)}$, so do $V_{j}^{t}$ and $V_{j}^{r(t)}$ on $E_{j}^{t} \cap E_{j}^{r(t)}$; so do, by (7.4), $V_{j}^{r(s)}$ and $V_{j}^{r(t)}$ on $E_{j}^{r(s)} \cap E_{j}^{r(t)}$. The latter contains $E_{j}^{s} \cap E_{j}^{t}$. (7.5) follows.

We can now define $\left(V_{j}^{\text {id }}\right)_{j=1}^{n}$. For any $\alpha \in \Gamma$, and $1 \leqq j \leqq n$, we take any $h \in C^{\text {id }}$ such that $\alpha \in E_{j}^{h} ; h_{j}=\alpha$ suffices for that. Then we define $V_{j}^{\text {id }}(\alpha):=V_{j}^{h}(\alpha)$. By (7.5), this does not depend on the particular choice of $h$; and every $V_{j}^{h}$ is now the restriction of $V_{j}^{\text {id }}$ to $E_{j}^{h}$.

For continuity, let $\sup \left(V_{j}^{\mathrm{id}}(\Gamma)\right)>\mu>v>\inf \left(V_{j}^{\text {id }}(\Gamma)\right)$. Openness of $\left\{f_{j}: \mu>\right.$ $\left.V_{j}^{\text {id }}\left(f_{j}\right)>v\right\}$ follows from Proposition 7.1, with $h$ such that $v>V_{j}^{\text {id }}\left(h_{j}\right)$ and, if $j>1, V_{j}^{\text {id }}\left(h_{j-1}\right)>\mu$. Continuity of $V_{j}$ follows.

Finally the uniqueness result. Any $\left(W_{j}^{\text {id }}\right)_{j=1}^{n}$, for which real $\tau_{j}, j=1, \ldots, n$, and positive $\sigma$ exist such that $W_{j}^{\text {id }}=\tau_{j}+\sigma V_{j}^{\text {id }}$ for all $j$, satisfy the requirements of the lemma. Conversely, if $\left(W_{j}^{\text {id }}\right)_{j=1}^{n}$ satisfy all the requirements of the lemma, then so do 


$$
Z_{j}^{\text {id. }}=\left[W_{j}^{\text {id }}-W_{j}^{\text {id }}\left(\beta^{0}\right)\right] /\left[W_{1}^{\text {id }}\left(\beta^{1}\right)-W_{1}^{\text {id }}\left(\beta^{0}\right)\right]
$$

From $Z_{j}^{\text {id }}\left(\beta^{0}\right)=0, Z_{1}^{\text {id }}\left(\beta^{1}\right)=1$, and from rereading the proof, the reader can see that this uniquely determines $Z_{j}^{\text {id }}, Z_{j}^{\text {id }}=V_{j}^{\text {id }}$ must hold for all $j$.

Note that we may not yet conclude that $\left(V_{j}^{\text {id }}\right)_{j=1}^{n}$ are additive value functions on all of $C^{\text {id }}$.

7.3. The functions $\left(V_{j}^{\mathrm{id}}\right)_{j=1}^{n}$ are additive value functions on $C^{\mathrm{id}}$

Lemma 7.6 For all (id-essential) $k: \quad[\alpha \geqslant \beta] \Leftrightarrow\left[V_{k}^{\text {id }}(\alpha) \geqslant V_{k}^{\text {id }}(\beta)\right]$. Hence $[\bar{\alpha} \geqslant \bar{\beta}] \Leftrightarrow\left[\sum V_{k}^{\mathrm{id}}(\alpha) \geqq \sum V_{k}^{\mathrm{id}}(\beta)\right]$.

Proof. Let $\alpha, \beta, k$ be arbitrary. Say $\alpha \geqslant \beta$. Let $f_{j}=\alpha$ for all $j<k, f_{j}=\beta$ for all $j \geqq k$. Then $(f=) f_{-k} \beta$ and $f_{-k} \alpha \in C^{\text {id }}$, and $f_{-k} \beta$ and $f_{-k} \alpha \in E^{f}$. By w.mon. and Lemma 7.5,

$$
[\alpha \approx \beta] \Rightarrow[\alpha \geqslant \beta \text { and } \beta \geqslant \alpha] \Rightarrow\left[f_{-k} \alpha \approx f_{-k} \beta\right] \Rightarrow V_{k}^{\text {id }}(\alpha)=V_{k}^{\text {id }}(\beta) \text {. }
$$

By com.s.mon. and Lemma 7.5,

$$
\alpha \succ \beta \Rightarrow f_{-k} \alpha \succ f_{-k} \beta \Rightarrow V_{k}^{\text {id }}(\alpha)>V_{k}^{\text {id }}(\beta)
$$

Analogously,

$$
\alpha<\beta \Rightarrow V_{k}^{\text {id }}(\alpha)<V_{k}^{\text {id }}(\beta)
$$

All of this together implies that $V_{k}^{\text {id }}$ represents $\succcurlyeq$ on $\Gamma$.

Lemma 7.7. Let $f \in C^{\mathrm{id}}, f \approx \bar{\alpha}$. Then $\sum_{j=1}^{n} V_{j}^{\mathrm{id}}\left(f_{j}\right)=\sum_{j=1}^{n} V_{j}^{\mathrm{id}}(\alpha)$.

Proof. The case $f_{j} \approx \alpha$ for all $j$ is direct. The case $f_{j} \succ \alpha$ for some $j$ and $f_{j} \prec \alpha$ for no $j$, and the case $f_{j} \prec \alpha$ for some $j$ and $f_{j} \succ \alpha$ for no $j$, are excluded by com.s.mon. So suppose $j<i$ exist such that $f_{j}>\alpha, f_{j+1} \approx \cdots \approx f_{i-1} \approx \alpha, f_{i}<\alpha$. We define $f^{0}$ such that $f_{k}^{0}=f_{k}$ for all $f_{k} \neq \alpha$, and $f_{k}^{0}=\alpha$ for all $f_{k} \approx \alpha$.

Now suppose, for some $0 \leqq l \leqq n-2, f^{l} \in C^{\text {id }}$ has been defined such that $f^{l} \approx \bar{\alpha}$, and $\sum V_{k}^{\text {id }}\left(f_{k}^{l}\right)=\sum V_{k}^{\text {id }}\left(f_{k}\right)$, with at least $l$ coordinates of $f^{l}$ equal to $\alpha$, and no coordinate of $f^{l}$ equivalent but unequal to $\alpha$. If in fact $f^{l}$ has $l+1$ or more coordinates equal to $\alpha$, define $f^{l+1}:=f^{l}$. If not, then, say:

$$
f_{m}^{l} \succ \alpha, \quad f_{a+1}^{l}=\cdots=f_{b-1}^{l}=\alpha, \quad f_{b}^{l} \prec \alpha, \quad \text { with } \quad b=a+l+1
$$


If now $f_{-a, b}^{l} \alpha, \alpha \approx \bar{\alpha}$, define $f^{l+1}:=f_{-a, b}^{l} \alpha, \alpha$.

If $f_{-a, b}^{l} \alpha, \alpha<\bar{\alpha}$, define, by Lemma $6.4, \alpha<f_{a}^{l+1} \preccurlyeq f_{a}^{l}$ such that

$$
f^{l+1}:=\left(f_{-b, a}^{l} \alpha, f_{a}^{l+1}\right) \approx \bar{\alpha} .
$$

If $f_{-a, b}^{l} \alpha, \alpha>\bar{\alpha}$, define $\alpha>f_{b}^{l+1} \geqslant f_{b}^{l}$ such that

$$
f^{l+1}:=\left(f_{-a, b}^{l} \alpha, f_{b}^{l+1}\right) \approx \bar{\alpha} .
$$

In any case, for $h=f_{-a}^{l} \alpha$, both $f^{l+1}$ and $f^{l}$ are in $E^{h}$, their ath coordinate being 'between' $f_{a-1}^{l}$ and $\alpha$, their $b$ th coordinate 'between' $\alpha$ and $f_{b}^{l}$. Hence by Lemma $7.5, f^{l}(\approx \bar{\alpha}) \approx f^{l+1}$ implies $\sum V_{k}^{\text {id }}\left(f_{k}^{l+1}\right)=\sum V_{k}^{\text {id }}\left(f_{k}^{l}\right)$.

Finally we end up with $f^{n-1} \approx \bar{\alpha}$, with $n-1$ coordinates equal to $\alpha$. Then by com.s.mon. the remaining coordinate of $f^{n-1}$ must also be equivalent, so equal, to $\alpha$. And

$$
\sum_{k} V_{k}^{\mathrm{id}}\left(f_{k}\right)=\sum_{k} V_{k}^{\mathrm{id}}\left(f_{k}^{0}\right)=\cdots=\sum_{k} V_{k}^{\mathrm{id}}\left(f_{k}^{n-1}\right)=\sum_{k} V_{k}^{\mathrm{id}}(\alpha)
$$

follows.

Theorem 7.1. There exists a continuous additive representation $V: f \mapsto$ $\sum_{j=1}^{n} V_{j}\left(f_{j}\right)$ for $\geqslant$ on $C^{\mathrm{id}}$, unique up to a positive affine transformation.

Proof. Let $f, g \in C^{\text {id }}$ be arbitrary. Let $\left(V_{j}^{\text {id }}\right)_{j=1}^{n}$ be as constructed above. Let $\alpha, \beta$ be such that $f \approx \bar{\alpha}, g \approx \bar{\beta}$ (Lemma 7.1). Then $f \geqslant g$ iff $\bar{\alpha} \geqslant \bar{\beta}$, which by Lemma 7.6 is iff $\sum_{k} V_{k}^{\text {id }}(\alpha) \geqq \sum_{k} V_{k}^{\text {idd }}(\beta)$. The latter by Lemma 7.7 holds iff $\sum_{k} V_{k}^{\text {id }}\left(f_{k}\right) \geqq \sum_{k} V_{k}^{\text {id }}\left(g_{k}\right)$.

\section{Completion of the proof of Theorem 5.1 under absence of maximal and minimal consequences}

Assumption 8.1 (for this section, with Theorem 8.1 excepted). The assumptions, and statement (ii), of Theorem 5.1 hold. There exists a $\pi$ with two $\pi$ essential states, say $\pi=$ identity. By $m$ we denote an id-essential state. No maximal or minimal consequences exist. Let $\beta^{1}>\beta^{0}$ be two fixed consequences. For every $\pi$ with two or more $\pi$-essential states, the continuous additive representation $V^{\pi}: f \mapsto \sum_{j=1}^{n} V_{j}^{\pi}\left(f_{j}\right)$ of $\geqslant$ on $C^{\pi}$ (which exists according to the previous section) is chosen such that $V_{j}^{\pi}\left(\beta^{0}\right)=0$ for all $j$, and $\sum_{j=1}^{n} V_{j}^{\pi}\left(\beta^{1}\right)=1$.

Note that we have changed 'scale', as compared to the previous section. 
There we had $V_{1}^{\text {id }}\left(\beta^{1}\right)=1$, now $\sum_{j=1}^{n} V_{j}^{\text {id }}\left(\beta^{1}\right)=1$. Note also that, at present, we may not yet conclude for different $\pi, \pi^{\prime}$, and $f \in C^{\pi}, g \in C^{\pi^{\prime}}$, that $f \geqslant g \Leftrightarrow \sum_{j=1}^{n} V_{j}^{\pi}\left(f_{j}\right) \geqq \sum_{j=1}^{n} V_{j}^{\pi^{\prime}}\left(g_{j}\right)$. The only consequences of Com.CCI that we used in the previous section, i.e., comonotonic coordinate independence, weak monotonicity, and comonotonic strong monotonicity, probably do not suffice for this purpose. We shall essentially use:

Lemma 8.1. Let there be at least two $\pi$-essential, and two $\pi^{\prime}$-essential, states. Let $k$ be $\pi^{\prime}$-essential. Then for all $l \in S, V_{l}^{\pi}=\phi_{l} \circ V_{k}^{\pi^{\prime}}$ on $C^{\pi} \cap C^{\pi^{\prime}}$ for a constant or positive affine $\phi_{l}$.

Proof. Say $\pi^{\prime}$ is the identity. We write $\phi$ for $\phi_{l}$. If $l$ is $\pi$-inessential, then $V_{l}^{\pi}$ is constant, and $\phi$ is the same constant. So assume:

$l$ is $\pi$-essential.

By Lemma 7.6 (which applies to all essential $k$ ) $V_{l}^{\pi}$ and $V_{k}^{\text {id }}$ represent the same $\succcurlyeq$, hence $V_{l}^{\pi}=\phi \circ V_{k}^{\text {id }}$ for an increasing $\phi$ which is continuous by Wakker (1986, Corollary VIII.10).

First note that Com.CCI implies the same property with all preferences replaced by equivalences. This we write out in terms of additive value functions, and with $\phi \circ V_{k}^{\text {id }}$ for $V_{l}^{\pi}$ everywhere:

$$
\begin{aligned}
& \text { for all } f_{-k} \alpha, g_{-k} \beta, f_{-k} \gamma, g_{-k} \delta \in C^{\text {id }} \text {, and } s_{-l} \alpha, t_{-l} \beta, s_{-l} \gamma, t_{-l} \delta \in C^{\pi}, \\
& \qquad\left\{V_{k}^{\text {id }}(\alpha)-V_{k}^{\text {id }}(\beta)={ }^{(1)} \sum_{j \neq k}\left[V_{j}^{\text {id }}\left(g_{j}\right)-V_{j}^{\text {id }}\left(f_{j}\right)\right]={ }^{(2)} V_{k}^{\text {id }}(\gamma)-V_{k}^{\text {id }}(\delta)\right\}
\end{aligned}
$$

and

$$
\phi \circ V_{k}^{\mathrm{id}}(\alpha)-\phi \circ V_{k}^{\mathrm{id}}(\beta)={ }^{(3)} \sum_{j \neq l}\left[V_{j}^{\pi}\left(t_{j}\right)-V_{j}^{\pi}\left(s_{j}\right)\right]
$$

imply

$$
\sum_{i \neq l}\left[V_{j}^{\pi}\left(t_{j}\right)-V_{j}^{\pi}\left(s_{j}\right)\right]={ }^{(4)} \phi \circ V_{k}^{\mathrm{id}}(\gamma)-\phi \circ V_{k}^{\mathrm{id}}(\delta)
$$

Now let $V_{k}^{\text {id }}(\mu)$ be an arbitrary element of $\operatorname{int}\left(V_{k}^{\text {id }}(\Gamma)\right)$. There can be seen to be an interval $S$ around $V_{k}^{\text {id }}(\mu)$, so small that for all $V_{k}^{\text {id }}(\alpha), V_{k}^{\text {id }}(\beta), V_{k}^{\text {id }}(\gamma)$, and $V_{k}^{\text {id }}(\delta) \in S$, there exist $f, g$ such that $f_{-k} \alpha, g_{-k} \beta, f_{-k} \gamma, g_{-k} \delta$ are in $C^{\text {id }}$, and such that $=^{(1)}$ is satisfied. For this we use the existence of a state $i \neq k$ which is id-essential, so that the interval $V_{i}^{\text {id }}(\Gamma)$ is non-degenerate. Of course, if $i<k$, then $f_{i} \geqslant \alpha, f_{i} \geqslant \gamma, g_{i} \geqslant \beta, g_{i} \geqslant \delta$ holds. If $i>k$, the reverse has to hold. Furthermore, by continuity of $\phi, S$ can be taken so small that $\phi(S)$ is small 
enough to guarantee existence of $s$ and $t$ such that $s_{-l} \alpha, t_{-l} \beta, s_{-l} \gamma, t_{-l} \delta$ are in $C^{\pi}$, and such that $=^{(3)}$ holds.

We conclude for all $V_{k}^{\text {id }}(\alpha), V_{k}^{\text {id }}(\beta), V_{k}^{\text {id }}(\gamma), V_{k}^{\text {id }}(\delta) \in S$,

$$
\begin{aligned}
& V_{k}^{\mathrm{id}}(\alpha)-V_{k}^{\mathrm{id}}(\beta)=V_{k}^{\mathrm{id}}(\gamma)-V_{k}^{\mathrm{id}}(\delta) \\
& \quad \Rightarrow \phi \circ V_{k}^{\mathrm{id}}(\alpha)-\phi \circ V_{k}^{\mathrm{id}}(\beta)=\phi \circ V_{k}^{\mathrm{id}}(\gamma)-\phi \circ V_{k}^{\mathrm{id}}(\delta) .
\end{aligned}
$$

This is now shown by choosing $f, g, s, t$ as above. (8.4), only for the case where $\beta=\gamma$, already suffices to show that on $S, \phi$ satisfies: $\phi\left(\left(\alpha^{*}+\delta^{*}\right) /\right.$ $\left.2)=\left[\phi\left(\alpha^{*}\right)+\phi\left(\delta^{*}\right)\right)\right] / 2$. By Theorem 1 of section 2.1.4 of Aczél (1966), or by (88) of section 3.7 of Hardy et al. (1934), $\phi$ must be affine on $S$. Hence it has constant derivative everywhere on int $\left(V_{l}(\Gamma)\right)$. Consequently $\phi$ must be affine on $V_{i}(\Gamma)$.

For all $\pi$ with two or more $\pi$-essential states, we can, by Lemma 8.1, and the fact that all $V_{j}^{\pi}\left(\beta^{0}\right)$ equal 0 , define $\lambda_{j}^{\pi} \in \mathbb{R}_{+}$such that, with $m$ id-essential:

$$
V_{j}^{\pi}=\lambda_{j}^{\pi} V_{m}^{\mathrm{id}}
$$

We define, for all these $\pi$,

$$
p_{j}^{n}:=\lambda_{j}^{\pi} / \sum_{i=1}^{n} \lambda_{i}^{\text {id }}
$$

For $\pi$ with exactly one $\pi$-essential state, say $l$, we define

$$
p_{l}^{\pi}:=1, p_{i}^{\pi}:=0 \text { for all } i \neq l
$$

Next we define $U: \Gamma \rightarrow \mathbb{R}$.

Definition 8.1. For all $\alpha \in \Gamma, U: \alpha \mapsto \sum_{j=1}^{n} V_{j}^{\mathrm{id}}(\alpha)$.

Lemma 8.2. For all $\pi$ with two or more $\pi$-essential states, and all $\alpha$, $V_{j}^{\pi}(\alpha)=p_{j}^{\pi} U(\alpha)$. For all $\pi, \sum p_{j}^{\pi}=1$.

Proof. Let $\pi$ have two $\pi$-essential states. Then

$$
\begin{aligned}
p_{j}^{\pi} U(\alpha) & =\left[\lambda_{j}^{\pi} / \sum_{i=1}^{n} \lambda_{i}^{\mathrm{id}}\right]\left[\sum_{i=1}^{n} V_{i}^{\mathrm{id}}(\alpha)\right] \\
& =\left[\lambda_{j}^{\pi} / \sum_{i=1}^{n} \lambda_{i}^{\mathrm{id}}\right]\left[\sum_{i=1}^{n} \lambda_{i}^{\mathrm{id}} V_{m}^{\mathrm{id}}(\alpha)\right]=V_{j}^{\pi}(\alpha) .
\end{aligned}
$$

For such $\pi, \sum p_{j}^{\pi}=\sum \lambda_{j}^{\pi} / \sum \lambda_{i}^{\text {id }}=\sum V_{j}^{\pi}\left(\beta^{1}\right) / \sum V_{i}^{\text {id }}\left(\beta^{1}\right)=1 / 1=1$.

For other $\pi$, with only one $\pi$-essential state, $\left[\sum p_{j}^{\pi}=1\right]$ is direct. 
Lemma 8.3. Let $f \in C^{\pi}, f \approx \bar{\alpha}$. Then $\sum p_{j}^{\pi} U\left(f_{j}\right)=\mathrm{U}(\alpha)$.

Proof. If there are two or more $\pi$-essential states, then by Lemma 7.7, adapted to $C^{\pi}, \sum V_{j}^{\pi}\left(f_{j}\right)=\sum V_{j}^{\pi}(\alpha)$. Hence then $\sum p_{j}^{\pi} U\left(f_{j}\right)=\sum p_{j}^{\pi} U(\alpha)=U(\alpha)$.

If $\pi$ has exactly one $\pi$-essential state, say $k$, then by Lemma $3.2, f \approx \bar{f}_{k}$. Hence by Lemma 7.6, $U\left(\bar{f}_{k}\right)=U(\alpha)$, i.e., $\sum p_{j}^{\pi} U\left(f_{j}\right)=U(\alpha)$.

Lemma 8.4. Let $f \in C^{\pi}, g \in C^{\pi^{\prime}}$. Then $f \geqslant g \Leftrightarrow \sum_{j=1}^{n} p_{j}^{\pi} U\left(f_{j}\right) \geqq \sum_{j=1}^{n} p_{j}^{\pi^{\prime}} U\left(g_{j}\right)$.

Proof. Let (Lemma 7.1) $f \approx \bar{\alpha}, g \approx \bar{\beta}$. Then $f \geqslant g$ iff $\bar{\alpha} \geqslant \bar{\beta}$, which by Lemma 7.6 holds iff $U(\alpha) \geqq U(\beta)$. By Lemma 8.3 the latter holds iff $\sum p_{j}^{\pi} U\left(f_{j}\right) \geqq$ $\sum p_{j}^{\pi} U\left(g_{j}\right)$

Lemma 8.5. Let $A \subset S . \quad$ Let $A=\{\pi(1), \ldots, \pi(k)\}=\left\{\pi^{\prime}(1), \ldots, \pi^{\prime}(k)\right\} . \quad$ Then $\sum_{j=1}^{k} p_{j}^{\pi}=\sum_{j=1}^{k} p_{j}^{\pi^{\prime}}$

Proof. Let $f_{j}=\beta^{1}$ for all $j \in A, f_{j}=\beta^{0}$ for all $j \notin A$. Then $f \in C^{\pi}$ and $f \in C^{\pi^{\prime}}$. Apply the above Lemma with $g=f$.

The following result holds in generality without Assumption 8.1 presumed.

Theorem 8.1. Let the assumptions of Theorem 5.1 hold. Let (ii) there hold. Furthermore, let no maximal or minimal consequences exist, and let there be a $\pi$ with two or more $\pi$-essential states. Then (i), and (5.1), of Theorem 5.1 hold.

Proof. According to Lemma 8.5, and (2.11), with $P^{\pi}(j):=p_{j}^{\pi}$ for all $\pi, j$, there exists a unique capacity $v$ in accordance with (2.6). Note that the $U$ in Definition 8.1 is continuous. Lemma 8.4 , and (2.7) now give (i) of Theorem 5.1.

To derive (5.1), say there are two id-essential states. Then the fact that $\left(P^{\text {id }}(j) U\right)_{j=1}^{n}$ are additive value functions for $\geqslant$ on $C^{\text {id }}$, and uniqueness up to a positive affine transformation of $x \mapsto \sum\left(V_{j}^{\text {id }}\right)\left(x_{j}\right)$ in Theorem 7.1, give uniqueness up to a positive affine transformation of $U$, and together with $\left[\sum P^{\text {id }}(j)=1\right]$ uniquely determine $\left(P^{\text {id }}(j)\right)_{j=1}^{n}$. Analogously $\left(P^{\pi}(j)\right)_{j=1}^{n}$ are uniquely determined for any $\pi$ with two or more $\pi$-essential states. If $\pi$ has exactly one $\pi$-essential state $k$, then $P^{\pi}(k)=1$ must hold, and $P^{\pi}(j)=0$ for all $j \neq k$.

\section{Maximal and/or minimal consequences}

In this section we derive the implication $(i i) \Rightarrow(i)$, and the uniqueness result (5.1) in Theorem 5.1, for the case where maximal and/or minimal consequences may exist. 
Assumption 9.1 (for this section). The assumptions of Theorem 5.1 hold. Also statement (ii) there holds. There exists a permutation $\pi$ with two or more $\pi$ essential states. We assume that $\pi$ is identity.

Lemma 9.1. Let $\alpha, \gamma \in \Gamma$ be such that $\alpha \succ \gamma$. Then there exists $\beta \in \Gamma$ such that $\alpha \succ \beta>\gamma$.

Proof. $E:=\{\beta: \beta>\gamma\}$ and $F:=\{\beta: \beta<\alpha\}$ are open and non-empty. Their union is $\Gamma$, for if $\delta \in F^{c}$ then $\delta \geqslant \alpha$ so $\delta>\gamma$. Hence by connectedness of $\Gamma, E$ and $F$ inust intersect.

Notation 9.1. $\Gamma^{*}:=\{\alpha \in \Gamma: \alpha$ is neither maximal nor minimal $\} . G^{\pi *}:=G^{\pi} \cap$ $\left(\Gamma^{*}\right)^{n}$

Since $\pi=$ id has a $\pi$-essential state, there exists $\alpha \succ \beta$. By Lemma 9.1, $\Gamma^{*}$ is non-empty, and has no ('new') maximal or minimal consequences itself.

Lemma 9.2. If $i$ is essential on $G^{\pi}$ (i.e. $\pi$-essential), then it is on $G^{\pi *}$.

Proof. Say $\pi$ is identity. There exist $\alpha, \beta \in \Gamma$ such that $\alpha>\beta$. Twice Lemma 9.1 gives $\gamma, \delta$ such that $\alpha>\gamma \succ \delta \succ \beta$. Let $f \in C^{\text {id }}$ have $f_{k}=\gamma$ for all $k \leqq i, f_{k}=\delta$

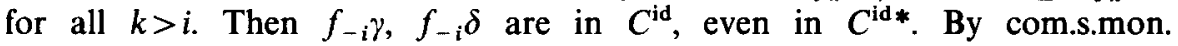
$f_{-i} \gamma>f_{-i} \delta$.

Next we show that, on $\left(\Gamma^{*}\right)^{n}$, (i) in Theorem 5.1 is satisfied.

Proposition 9.1. There exist a capacity $v$, and a continuous function $U^{*}: \Gamma^{*} \rightarrow \mathbb{R}$, such that $f \mapsto \int\left(U^{*} \circ f\right) \mathrm{d} v$ represents $\succcurlyeq$ on $\left(\Gamma^{*}\right)^{n}$.

Proof. By Lemma 9.1, $\Gamma^{*}$ itself has no maximal or minimal consequences. By Lemma 9.2, essentiality of states on $\left(\Gamma^{*}\right)^{n}$ is as on $\Gamma^{n}$. The proposition now follows from Theorem 8.1, if the required topological conditions can be guaranteed. This is done analogously to subsection 7.1: $\tau(\succcurlyeq) \mid \Gamma^{*}$ is taken as topology on $\Gamma^{*}$. Mainly by Lemma 7.2 and an analogue of Lemma 7.3, connectedness and separability are preserved. Continuity of $\geqslant$ on $\left(\Gamma^{*}\right)^{n}$ with respect to the product topology of the $\tau(\geqslant) \mid \Gamma^{*}$ 's, differs only in details from Lemma 7.4.

Lemma 9.3. If $\alpha$ is maximal [respectively minimal], then $U^{*}\left(\Gamma^{*}\right)$ is bounded above [respectively below].

Proof. The proof is given only for the case of a maximal $\alpha$. Let $i<j$ be two id-essential states. Let, only in this proof, $(\bar{\beta}, \bar{\gamma})$ denote the act $h$ with $h_{k}=\beta$ for $k \leqq i, h_{k}=\gamma$ for $k>i$, for all $\beta, \gamma \in \Gamma$. By com.s.mon., for all $\gamma \in \Gamma^{*}, \bar{\alpha}>(\bar{\alpha}, \bar{\gamma})$. 
Let $\gamma \in \Gamma^{*}$ be fixed, let $\beta$ (by Lemma 7.1 ) be such that $(\bar{\alpha}, \bar{\gamma}) \approx \bar{\beta}$ (so $\beta \in \Gamma^{*}$ ). Now for all $\mu \in \Gamma^{*}$ with $\mu \geqslant \gamma,(\bar{\mu}, \bar{\gamma})$ is in $\Gamma^{\text {ld } *}$, and $(\bar{\mu}, \bar{\gamma})<(\bar{\alpha}, \bar{\gamma}) \approx \bar{\beta}$, so

$$
v(\{1, \ldots, i\}) U^{*}(\mu)+[v(S)-v(\{1, \ldots, i\})] U^{*}(\gamma)<\mathrm{U}^{*}(\beta) .
$$

Since $i$ is essential on $\Gamma^{i d *}, v(\{1, \ldots, i\})$ is positive, and (9.1) induces an upper bound for $\left\{U^{*}(\mu): \mu \in \Gamma^{*}, \mu \succcurlyeq \gamma\right\}$, thus for $U^{*}\left(\Gamma^{*}\right)$.

Definition 9.1. If $\alpha \in \Gamma$ is maximal, then $U(\alpha):=\sup \left(U^{*}\left(\Gamma^{*}\right)\right)$. If $\alpha \in \Gamma$ is minimal, then $U(\alpha):=\inf \left(U^{*}\left(\Gamma^{*}\right)\right)$. If $\alpha \in \Gamma^{*}$, then $U(\alpha):=U^{*}(\alpha)$.

As we saw above, $U(\alpha) \in \mathbb{R}$ for all $\alpha$.

Notation 9.2. $\Gamma^{+}:=\Gamma^{*} \cup\{\alpha \in \Gamma: \alpha$ is maximal $\}$.

Lemma 9.4. For all $f \in\left(\Gamma^{+}\right)^{n}$, and $\theta \in \Gamma$ with $f \approx \bar{\theta}, \int(U \circ f) \mathrm{d} v=U(\theta)$.

Proof. Say $f \in \Gamma^{\mathrm{id}}$. By com.s.mon., $\theta$ is not minimal, so $\theta \in \Gamma^{+}$. If no maximal $\alpha$ exists, Proposition 9.1 gives the desired result. So let $\alpha$ be maximal. Let $0 \leqq k \leqq n$ be such that $f_{1} \approx \alpha, \ldots, f_{k} \approx \alpha, f_{k+1} \prec \alpha, \ldots, f_{n} \prec \alpha$. If $\theta$ is maximal, then $\theta \approx \alpha$, and by com.s.mon. $k+1, \ldots, n$ must be id-inessential. Then $\int(U \circ f) \mathrm{d} v=U(\theta)$ follows.

There remains the most complicated case, where $\theta$ is not maximal, so, neither being minimal, is in $\Gamma^{*}$. First we show that $\int(U \circ f) \mathrm{d} v \leqq U(\theta)$. By w.mon., for all $\mu \in \Gamma^{*}$ with $(\alpha \succ) \mu \geqslant f_{k+1}$,

$$
\left(\mu, \ldots, \mu, f_{k+1}, \ldots, f_{n}\right) \preccurlyeq \theta, \quad \text { i.e., } \quad \int\left(U \circ\left(\mu, \ldots, \mu, f_{k+1}, \ldots, f_{n}\right)\right) \mathrm{d} v \leqq U(\theta) .
$$

Writing, for all $1 \leqq j \leqq k, U\left(f_{j}\right)=U(\alpha)=\sup \left\{U(\mu): \mu \in \Gamma^{*}, \mu \succcurlyeq f_{k+1}\right\}$ shows that $\int(U \circ f) \mathrm{d} v \leqq U(\theta)$.

To see that $\int(U \circ f) \mathrm{d} v \geqq U(\theta)$, we consider $\delta$ such that $\theta \succ \delta$, so $f \succ \bar{\delta}$. By standard arguments continuity of $\geqslant$, and connectedness of $\Gamma$, imply existence of $\mu_{k}$ such that $f_{k} \succ \mu_{k} \succcurlyeq f_{k+1}$, and $f_{-k} \mu_{k}>\delta$. Also, $\mu_{k-1}$ exists such that $f_{k-1}>\mu_{k-1} \geqslant \mu_{k}$ and $\left(f_{-k-1, k} \mu_{k-1}, \mu_{k}\right)>\delta$. Finally we end up with $\alpha>\mu_{1} \geqslant$ $\mu_{2} \geqslant \cdots \geqslant \mu_{k}$ such that $\left(\mu_{1}, \ldots, \mu_{k}, f_{k+1}, \ldots, f_{n}\right)>\bar{\delta}$. Hence, for all $\mu \in \Gamma$ such that $\alpha>\mu \geqslant \mu_{1}\left(\geqslant \cdots \geqslant \mu_{k}\right)$, we obtain $\int\left(U \circ\left(\mu, \ldots, \mu, f_{k+1}, \ldots, f_{n}\right)\right) \mathrm{d} v>U(\delta)$.

Substituting, for $1 \leqq j \leqq k, U\left(f_{j}\right)=U(\alpha)=\sup \left\{U(\mu): \mu \in \Gamma^{*}, \mu \geqslant \mu_{1}\right\}$, shows that $\int(U \circ f) \mathrm{d} v \geqq U(\delta)$. This holds for all $\delta<\theta$. Hence $\int(U \circ f) \mathrm{d} v \geqq U(\theta)$.

Lemma 9.5. The map $f \mapsto \int(U \circ f) \mathrm{d} v$ represents $\geqslant$ on $\left(\Gamma^{+}\right)^{n}$.

Proof. First for constant acts. Suppose $\gamma>\delta$, with $\gamma$ maximal. Then, by 
Lemma 9.1, $\gamma>\alpha \succ \delta$ for some $\alpha \in \Gamma$. So $U(\gamma) \geqq U(\alpha)>U(\delta)$ follows, the latter strict inequality follows from Proposition 9.1. All other cases of $\gamma \geqslant \delta \Leftrightarrow U(\gamma) \geqq U(\delta)$ are straightforward.

Next let $f, g \in\left(\Gamma^{+}\right)^{n}$ be arbitrary. Let $f \approx \bar{\gamma}, g \approx \bar{\delta}$ (by Lemma 7.1). Then $f \geqslant g \Leftrightarrow \bar{\gamma} \geqslant \bar{\delta} \Leftrightarrow U(\gamma) \geqq U(\delta) \Leftrightarrow \int(U \circ f) \mathrm{d} v \geqq \int(U \circ g) \mathrm{d} v$, the latter by Lemma 9.4.

Next we must turn to $\left(\Gamma^{+} \cup\{\alpha \in \Gamma: \alpha \text { is minimal }\}\right)^{n}=\Gamma^{n}$, and show that also here $f \mapsto \int(U \circ f) \mathrm{d} v$ represents $\geqslant$. This is very analogous to the above, elaboration is left out. We conclude that the implication (ii) $\Rightarrow$ (i) in Theorem 5.1 is now also established if maximal and/or minimal consequences exist. For the uniqueness result (5.1) in Theorem 5.1, we must show that for maximal [respectively minimal] $\alpha$ no other choice for $U(\alpha)$, than $\sup \left(U\left(\Gamma^{*}\right)\right)$ [or $\left.\inf \left(U\left(\Gamma^{*}\right)\right)\right]$ can be made. This can be seen for instance from the proof of Lemma 9.4. Let $i>j$ be two id-essential states. Then, with $\alpha$ maximal, $f_{1}=\cdots=f_{i}=\alpha, \quad \alpha \succ f_{i+1} \geqslant \cdots \geqslant f_{n}$, the formula $f(U \circ f) \mathrm{d} v=U(\theta)$ there uniquely determines $U(\alpha)$. For minimal consequences matters are analogous.

\section{Survey of the proof of Theorem 5.1, and concluding remarks}

The implication (i) $\Rightarrow$ (ii) in Theorem 5.1 has been established directly below the theorem. The proof of (ii) $\Rightarrow$ (i) for the case where no $\pi$ has two or more $\pi$-essential states, and the proof of the uniqueness results (5.2) and (5.3), have been given in Lemma 6.3. There remains the case where at least one $\pi$ has two or more $\pi$-essential states. The case of no maximal or minimal consequences is handled in Theorem 8.1, the existence of maximal consequences is handled in Lemma 9.5, the general case in the final lines of section 9.

Topological separability of $\Gamma$ is needed only for permutations $\pi$ with exactly one $\pi$-essential state. This is shown in Wakker (1986, ch. VI).

Let us now shortly and somewhat incompletely sketch how in the set-up of Schmeidler (1984a) the conditions of 'comonotonic independence' and w.mon. (called 'monotonicity' by Schmeidler) in the presence of the other 'usual' conditions, imply Com.CCI. Schmeidler assumes that $\Gamma$ is a set of lotteries, so that a mixture operation can be defined in the usual way. Then von Neumann-Morgenstern independence requires the implication

$$
f>g \text { and } 0<\lambda \leqq 1 \Rightarrow \lambda f+(1-\lambda) h>\lambda g+(1-\lambda) h
$$

for any $f, g, h$. Schmeidler weakens this to comonotonic independence by requiring the implication only for comonotonic $\{f, g, h\}$, and uses mainly this to characterize maximization of a Choquet-integral. Now suppose further that $f_{-i} \alpha, g_{-i} \beta, f_{-i} \gamma, g_{-i} \delta, s_{-j} \alpha, t_{-j} \beta, s_{-j} \gamma, t_{-j} \delta$ are as in Definition 4.1, 
with the exception that now $s_{-j} \gamma \prec t_{-j} \delta$, in violation of Com.CCI. Let us for simplicity suppose furthermore that all preferences in Definition 4.1 are strict (the general case is derived from this through the continuity condition in Schmeidler's set-up, and mixtures with better/worse constant acts, with weights tending to zero). A violation of w.mon. will be derived. We get

$$
\frac{1}{2}\left(f_{-i} \alpha\right)+\frac{1}{2}\left(g_{-i} \delta\right)<\frac{1}{2}\left(g_{-i} \beta\right)+\frac{1}{2}\left(g_{-i} \delta\right)<\frac{1}{2}\left(g_{-i} \beta\right)+\frac{1}{2}\left(f_{-i} \gamma\right)
$$

the first preference by $f_{-i} \alpha<g_{-i} \beta$ and comonotonic independence, the second preference by $f_{-t} \gamma>g_{-i} \delta$ and comonotonic independence. Further

$$
\frac{1}{2}\left(s_{-j} \alpha\right)+\frac{1}{2}\left(t_{-j} \delta\right) \succ \frac{1}{2}\left(t_{-j} \beta\right)+\frac{1}{2}\left(t_{-j} \delta\right) \succ \frac{1}{2}\left(t_{-j} \beta\right)+\frac{1}{2}\left(s_{-j} \gamma\right)
$$

the first preference by $s_{-j} \alpha \succ t_{-j} \beta$ and comonotonic independence, the second by $s_{-j} \gamma<t_{-j} \delta$ and comonotonic independence. Deleting middle mixtures in the above triples, and rewriting, we get

$$
\left[\frac{1}{2} f+\frac{1}{2} g\right]_{-i}\left[\frac{1}{2} \alpha+\frac{1}{2} \delta\right]<\left[\frac{1}{2} f+\frac{1}{2} g\right]_{-i}\left[\frac{1}{2} \beta+\frac{1}{2} \gamma\right]
$$

and

$$
\left[\frac{1}{2} s+\frac{1}{2} t\right]_{-j}\left[\frac{1}{2} \alpha+\frac{1}{2} \delta\right]>\left[\frac{1}{2} s+\frac{1}{2} t\right]_{-j}\left[\frac{1}{2} \beta+\frac{1}{2} \gamma\right] .
$$

If $\left[\frac{1}{2} \alpha+\frac{1}{2} \delta\right] \geqslant\left[\frac{1}{2} \beta+\frac{1}{2} \gamma\right]$, then the first preference violates w.mon, if $\left[\frac{1}{2} \alpha+\frac{1}{2} \delta\right] \preccurlyeq$ $\left[\frac{1}{2} \beta+\frac{1}{2} \gamma\right]$ then the second preference violates w.mon.

The main result of this paper, Theorem 5.1, provides, under restrictions appropriate for economic applications, a behavioural foundation for nonadditive probabilities in decision making under uncertainty. It is hoped that this will help in clarifying the usefulness of non-additive probabilities.

\section{References}

Aczél, J., 1966, Lectures on functional equations and their applications (Academic Press, New York).

Anscombe, F.J. and R.J. Aumann, 1963, A definition of subjective probability, Annals of Mathematical Statistics 34, 199-205.

Choquet, G., 1953-54, Theory of capacities, Annales de l'Institut Fourier (Grenoble), 131-295.

Debreu, G., 1960, Topological methods in cardinal utility theory, in: K.J. Arrow, S. Karlin and P. Suppes, eds., 1959, Mathematical methods in the social sciences (Stanford University Press, Stanford, CA) 16-26.

Debreu, G., 1964, Continuity properties of Paretian utility, International Economic Review 5, 285-293.

Dellacherie, C., 1970, Quelques commentaires sur les prolongements de capacités, Seminaire de Probabilités V Strasbourg, Lecture Notes in Mathematics, no. 191 (Springer, Berlin).

Gilboa, I., 1987, Expected utility with purely subjective non-additive probabilities, Journal of Mathematical Economics 16, 65-88.

Hardy, G.H., J.E. Littlewood and G. Pólya, 1934, Inequalities (Cambridge University Press, Cambridge). 
Krantz, D.H., R.D. Luce, P. Suppes and A. Tversky, 1971, Foundations of measurement, Vol. I: Additive and polynomial representations (Academic Press, New York).

Savage, L.J., 1954, The foundations of statistics (Wiley, New York).

Schmeidler, D., 1984a, Subjective probability and expected utility without additivity, Caress working paper 84-21 (first part) (Center for Analytic Research in Economics and the Social Sciences, University of Pennsylvania, Philadelphia, PA).

Schmeidler, D., 1984b, Nonadditive probabilities and convex games, Caress working paper 84 21 (second part) (Center for Analytic Research in Economics and the Social Sciences, University of Pennsylvania, Philadelphia, PA).

Schmeidler, D., 1986, Integral representation without additivity, Proceedings of the American Mathematical Society 97, 255-261.

Wakker, P.P., 1984, Cardinal coordinate independence for expected utility, Journal of Mathematical Psychology 28, 110-117.

Wakker, P.P., 1986, Representations of choice situations, Ph.D. dissertation (Department of Economics, University of Brabant, Tilburg).

Wakker, P.P., 1987, Nonadditive probabilities and derived strengths of preferences, Internal report 87 MA 03 (Department of Mathematical Psychology, University of Nijmegen, Nijmegen). 